\title{
Adsorption de la quinine bichlorhydrate sur un charbon actif peu coûteux à base de la Bagasse de canne à sucre imprégnée de l'acide phosphorique
}

\author{
Anatole KIFUANI KIA MAYEKO ${ }^{1, *}$, Philippe NOKI VESITULUTA ${ }^{1}$, \\ Josaphat NDELO DI PHANZU ${ }^{2}$, Daniel MUKANA WA MUANDA ${ }^{1}$, \\ Gracien EKOKO BAKAMBO ${ }^{1}$, Bernard ILINGA LOPAKA ${ }^{1}$ et \\ Joseph MUKINAYI MULANGALA ${ }^{1}$
}

${ }^{1}$ Laboratoire de Chimie Organique Physique, Eau et Environnement (LACOPE), Université de Kinshasa, Faculté des Sciences, B.P.190 Kinshasa XI, R.D.Congo.

${ }^{2}$ Laboratoire de Toxicologie, Université de Kinshasa, Faculté des Sciences Pharmaceutiques, R.D.Congo. *Corresponding author, E-mail: kifuanianatole@yahoo.fr; Tel:243 998229987

\section{RESUME}

L'adsorption de la Quinine Bichlorhydrate (QBC) sur charbon actif a été étudiée en réacteur discontinu. Le charbon actif (BP) utilisé a été préparé par imprégnation de la Bagasse de canne à sucre dans une solution de $\mathrm{H}_{3} \mathrm{PO}_{4}$ et activation thermique à $900{ }^{\circ} \mathrm{C}$, pendant une heure, en absence des gaz activants. L'image SEM obtenue montre une porosité très développée. Les images TEM et XRD montrent l'absence de cristallisation dans ce charbon. La surface spécifique est de $1439,46 \mathrm{~m}^{2} / \mathrm{g}$. Le charbon actif est acide (pHcontact 6,2), son $\mathrm{pH}_{\mathrm{ZPC}}$ est 6,2. Il présente des fonctions oxygénées de surface, déterminées par la méthode de Boehm et par FT-IR. L'imprégnation avec l'acide phosphorique développe mieux les fonctions acides carboxyliques comparées aux fonctions lactone ou phénol. Les modèles de diffusion intraparticulaire et d'Elovich sont bien appropriés pour décrire l'adsorption de QBC sur charbon actif BP comparés aux modèles de pseudo-ordre 1 et 2 . Les isothermes d'adsorption sont essentiellement de type $\mathrm{S}$ et indiquent une adsorption multicouches. L'isotherme de type $\mathrm{L}$ est obtenue à $\mathrm{pH}$ 2,00. Les isothermes obtenues suivent les modèles de Freundlich et Langmuir. La constante d'affinité $\mathrm{K}_{\mathrm{L}}$ augmente de $0,383 \cdot 10^{5} \mathrm{~L} \cdot \mathrm{mol}^{-1}$ à $1,902 \cdot 10^{5} \mathrm{~L} \cdot \mathrm{mol}^{-1}$, lorsque le $\mathrm{pH}$ passe de 1,05 à 5,82 . Le paramètre d'équilibre $\mathrm{R}_{\mathrm{L}}$ et le paramètre $1 / \mathrm{n}$ inférieurs à 1 , indiquent que l'adsorption de QBC sur charbon actif BP est favorable.

(C) 2012 International Formulae Group. All rights reserved.

Mots clés: Charbon actif, quinine bichlorhydrate, bagasse de canne à sucre, cinétique, isotherme, imprégnation.

\section{INTRODUCTION}

L'utilisation accrue de certains produits chimiques d'origine industrielle (colorants, détergents, hydrocarbures, phénols, engrais, pesticides) ou médicale (antipaludéens, antibiotiques, analgésiques, etc.), est à la base de nombreux cas de pollution des eaux, des sols, de l'air ou d'intoxications accidentelles ou volontaires, et, suscite à l'heure actuelle une attention particulière, à l'échelle mondiale. On assiste ainsi aujourd'hui de plus en plus à de nombreux cas d'altérations des 
écosystèmes et à des problèmes de santé publique pouvant conduire à l'élimination des espèces vivantes (végétaux, animaux, hommes).

De nombreux scientifiques des différentes disciplines s'intéressent, de plus en plus, à l'identification et à l'élimination des polluants de l'environnement ou des toxiques à la base de la morbidité et mortalité chez l'homme ou chez les animaux. A cet effet, on recourt souvent à l'adsorption sur charbon actif, notamment pour l'élimination des toxiques, des micropolluants organiques ou inorganiques de l'eau, la décoloration des huiles végétales et la purification de nombreux produits (jus, sirop de sucrerie, vin, bière, etc.) (Kifuani et al., 2004 ; Muhammad et Muhammad, 2007 ; Maurya et al., 2008 ; Khlelifi et al., 2009 ; Schimmel et al., 2010). Le charbon actif est aussi employé dans la catalyse hétérogène comme support des catalyseurs ou comme catalyseur (Quintanilla et al., 2008 ; Padhye et al., 2010 ; Lin et al., 2011).

L'adsorption des composés organiques sur charbon actif est principalement influencée par les caractéristiques de l'adsorbat (taille, charge, polarité, aromaticité, concentration, etc.), de l'adsorbant (porosité, surface spécifique, volume de pores, charge de surface, fonctions de surface, hydrophilie, hydrophobie, etc.) et du milieu ( $\mathrm{pH}$, force ionique, présence d'autres molécules adsorbables, température). De nombreux travaux rapportent divers procédés qui utilisent une diversité des matériaux précurseurs pour la préparation du charbon actif. La détermination des caractéristiques et des propriétés adsorbantes du charbon actif fait aussi l'objet de plusieurs travaux rapportés dans la littérature (Avom et al., 2001; Arivoli et al., 2007 ; Laximi Gayatri et al., 2010). En effet, on peut utiliser toute matière carbonée pour la préparation du charbon actif.
Les antipaludéens (quinine, amodiaquine, méfloquine, chloroquine, etc.) se sont révélés être parmi les médicaments pris à grande échelle dans les pays en développement ou l'incidence du paludisme est très élevée. Ces produits entraînent plusieurs cas d'intoxication chez l'homme. Le charbon médicinal n'est pas toujours disponible dans les hôpitaux des pays en développement, et, de nombreux cas d'intoxication médicamenteuse ou chimique restent sans solution appropriée.

L'objet de ce travail est, dans un premier temps, de préparer un charbon actif à partir des déchets des sciures de la Bagasse de canne à sucre, de déterminer ses propriétés texturales, physiques et chimiques, ses caractéristiques de conformité aux exigences des normes de la pharmacopée. Dans un deuxième temps, d'étudier l'adsorption d'un composé organique modèle, la Quinine Bichlorhydrate sur le charbon actif préparé. Une étude des isothermes d'adsorption et cinétiques en fonction du $\mathrm{pH}$ est effectuée pour élucider le mécanisme d'adsorption de la Quinine Bichlorhydrate sur ce charbon actif.

\section{MATERIEL ET METHODES \\ Préparation du charbon actif \\ Traitement préliminaire du matériau précurseur}

La Bagasse de canne à sucre utilisée dans cette étude a été obtenue à la Compagnie Sucrière de Kwilu-Ngongo, dans la province du Bas-Congo (R.D. Congo). Le traitement préliminaire du matériau précurseur a consisté non seulement à le débarrasser des phases cristallines (quartz), mais aussi à le réduire à une même frange de dimension granulométrique ( $\leq 1 \mathrm{~mm})$. La sciure de la Bagasse a été obtenue par broyage de ce matériau végétal dans un moulin (FRITSCH INDUSTRIMEST) muni d'un tamis de mailles de $1 \mathrm{~mm}$ de diamètre. La sciure ainsi obtenue a été séchée pendant sept jours à la température du laboratoire, puis à l'étuve 
(DESPATCH Oven Co de type Elect) à $45^{\circ} \mathrm{C}$, pendant sept jours.

Préparation de la solution d'imprégnation et imprégnation des sciures

La solution de $\mathrm{H}_{3} \mathrm{PO}_{4}$ utilisée pour l'imprégnation des sciures a été préparée à partir d'une solution commerciale de $\mathrm{H}_{3} \mathrm{PO}_{4}$ (MERCK, p.a. 85\%, d 1,69). La solution d'imprégnation a été préparée par dilution d'un volume requis de la solution commerciale dans un ballon jaugé en verre. La solution préparée est gardée à l'abri de la lumière dans un flacon en verre borosilicaté. L'imprégnation des sciures a été faite en plaçant la masse des sciures dans un imprégneur hermétique (LACOP-IP M100) contenant la solution d'imprégnation.

\section{Carbonisation et activation}

La carbonisation des sciures imprégnées a été conduite dans un four (NABER, modèle $\mathrm{N} 7 / \mathrm{H}$ ), à $500 \pm 2{ }^{\circ} \mathrm{C}$, pendant trente minutes, en utilisant des pyrolyseurs (LACOP-PCA, modèle 2M1). L'activation thermique des carbonisats a été effectuée en plaçant les pyrolyseurs dans le four, en absence des gaz activants classiques $\left(\mathrm{CO}_{2}\right.$, $\mathrm{H}_{2} \mathrm{O}, \mathrm{O}_{2}$, etc.). La température d'activation était de $900{ }^{\circ} \mathrm{C}$ et la durée de l'activation d'une heure. Les sciures de la Bagasse non imprégnées ont aussi été carbonisées et activées thermiquement dans les mêmes conditions que les sciures imprégnées.

\section{Traitement final}

Le charbon obtenu après carbonisation et activation thermique est lavé avec une solution normale d'acide chlorhydrique pour éliminer les traces d'agent d'imprégnation résiduel et les oxydes métalliques formés au cours de la carbonisation, à partir des métaux naturellement présents dans les matériaux végétaux utilisés. Ce traitement permet aussi de débarrasser le charbon d'éventuels traces d'agent d'imprégnation. L'acide chlorhydrique est éliminé par lavage avec de l'eau distillée. Le lavage est suivi par des tests de coloration au méthylorange $1 \%$. Le charbon lavé est séché et tamisé. Les études ultérieures ont été effectuées avec le charbon actif en poudre (CAP) ainsi obtenu et noté $\mathrm{BP}$ pour le charbon actif obtenu avec une imprégnation dans l'acide phosphorique et B0 pour le charbon actif obtenu sans imprégnation préalable.

\section{Caractérisation du charbon actif Détermination des propriétés texturales et de la composition élémentaire}

Les propriétés texturales du charbon actif ont été déterminées par microscopie électronique à balayage (SEM, Scanning electronic microscopy). Nous avons utilisé un microscope électronique JEOL JSM - $6700 \mathrm{~F}$ à effet de champ (Fe-SEM, Field emission scanning electron microscope) équipé d'un spectromètre électronique des rayons $\mathrm{X}$ en dispersion d'énergie (EDS, Energy-dispersive $\mathrm{X}$-ray spectrophotometer). L'EDS a été utilisé en mode semi-quantitatif et a permis la détermination de la composition élémentaire du charbon actif.

\section{Etat amorphe du charbon actif}

La microscopie électronique à transmission (TEM, Transmission electronic microscopy) et la diffraction électronique aux rayons X (XRD, X-ray diffraction) ont été utilisées pour déterminer la structure amorphe du matériau préparé. Nous avons utilisé un microscope TEM conventionnel (JEOL JEM200CX, 200kV). L'analyse XRD a été réalisée à l'aide d'un diffractomètre de rayons X (DMAX- R3) comprenant un monochromateur arrière permettant d'éliminer la fluorescence $\mathrm{du}$ fer, et travaillant sur la radiation monochromatique du cuivre, $\mathrm{K} \alpha 1$ $(1,54178 \dot{\mathrm{A}})$. Le domaine observé de l'angle $2 \theta$ est compris entre $2^{\circ}$ et $80^{\circ}$.

\section{Surface spécifique}

La surface spécifique a été déterminée par la méthode d'adsorption de bleu de méthylène (MBAM, methylene blue adsorption method). A cet effet, $50 \mathrm{~mL}$ de solution de Bleu de méthylène (BM) $28.10^{-6}$ $\mathrm{M}\left(\mathrm{C}_{0}\right)$ sont mis en contact avec $1 \mathrm{mg}$ de Charbon actif $\left(\mathrm{m}_{\mathrm{C}}\right)$. La suspension est agitée 
jusqu'à l'équilibre. Des prélèvements effectués toutes les dix minutes permettent de déterminer le temps d'équilibre et donc la concentration à l'équilibre $\left(\mathrm{C}_{\mathrm{e}}\right)$ de la solution résiduelle de $\mathrm{BM}$ obtenue après centrifugation, par mesure de la densité optique à $\lambda_{\max }$. On calcule alors $\mathrm{Q}_{\mathrm{m}}$, la capacité maximale d'adsorption du charbon actif pour BM à l'aide de la relation (1).

$$
\mathrm{Q}_{\mathrm{m}}=\frac{\left(\mathrm{C}_{\mathrm{o}}-\mathrm{C}_{\mathrm{e}}\right) \cdot \mathrm{V}}{\mathrm{m}_{\mathrm{C}}}
$$

Avec : $\mathrm{Q}_{\mathrm{m}}$, la capacité maximale d'adsorption $\mathrm{du}$ charbon pour BM $\left(\mathrm{mol}^{-\mathrm{g}^{-1}}\right) ; \mathrm{C}_{\mathrm{o}}$, la concentration initiale de la solution de BM $\left(\operatorname{mol} . \mathrm{L}^{-1}\right) ; \mathrm{C}_{\mathrm{e}}$, la concentration à l'équilibre de la solution de BM $\left(\operatorname{mol} . \mathrm{L}^{-1}\right) ; \mathrm{m}_{\mathrm{C}}$, la masse $\mathrm{du}$ charbon actif $(\mathrm{g})$; $\mathrm{V}$, le volume de la solution $(\mathrm{mL})$. La surface spécifique $\mathrm{S}_{\mathrm{BM}}$ (déterminée à l'aide de $\mathrm{BM}$ ) est calculée à partir de l'équation suivante :

$\mathrm{S}_{\mathrm{BM}}=\mathrm{Q}_{\mathrm{m}} \cdot \mathrm{N}_{\mathrm{A}} \cdot \mathrm{s} \quad(2)$

Avec: $S_{B M}$, la surface spécifique déterminée en utilisant $\mathrm{BM}$ comme adsorbat $\left(\mathrm{m}^{2} / \mathrm{g}\right) ; \mathrm{Q}_{\mathrm{m}}$, la capacité maximale d'adsorption $(\mathrm{mol} / \mathrm{g})$; $\mathrm{s}$, l'aire occupée par une molécule de BM $\left(175 \dot{\mathrm{A}}^{2}\right.$ ) (Adamson, 1982) et $\mathrm{N}_{\mathrm{A}}$, le nombre d'Avogadro.

\section{Détermination des fonctions chimiques de surface}

Les fonctions chimiques de surface ont été déterminées par dosage selon la méthode de Boehm (1966) et par spectrophotométrie infrarouge.

\section{Méthode de Boehm}

La méthode de Boehm (1966) permet une évaluation quantitative des fonctions acides et basiques de surface du charbon actif. A cet effet, $1 \mathrm{~g}$ de charbon actif, préalablement séché à l'étuve à $110{ }^{\circ} \mathrm{C}$, est mis en contact dans un Adsorbeur (LACOP ADS $X-2$ ) pendant 72 heures, sous agitation, avec $50 \mathrm{~mL}$ d'une solution $0,1 \mathrm{~N}$ de l'une des trois bases suivantes: $\mathrm{NaHCO}_{3}, \mathrm{Na}_{2} \mathrm{CO}_{3}$ et $\mathrm{NaOH}$, permettant de classer les groupements de surface de type acide, en acide carboxylique $(-\mathrm{COOH})$, lactone $(-\mathrm{COO})$ ou phénol $(-\mathrm{OH})$. Le traitement de cette masse de charbon actif avec une solution décinormale d'acide chlorhydrique permet de classer les groupements de surface de type basique. Le volume réactionnel de base ou d'acide est déterminé par titrage en retour avec une solution normale d'acide chlorhydrique ou d'hydroxyde de sodium, respectivement pour les fonctions de surface de type acide et basique.

\section{Méthode FT - IR}

L'infrarouge à transformée de Fourrier (FTIR, Fourrier Transform infrared spectroscopy) est un outil important permettant la détermination qualitative détaillée des fonctions chimiques de surface (Mangun, 1999). Les échantillons à analyser ont été préparés sur des pastilles de bromure de potassium $(\mathrm{KBr})$ dans les proportions de $300 \mathrm{mg}$ de $\mathrm{KBr}$ et $5 \mathrm{mg}$ de matériau finement broyé. L'analyse des pastilles a été réalisée, en balayant les longueurs d'onde de $400 \mathrm{~cm}^{-1}$ à $3500 \mathrm{~cm}^{-1}$, à l'aide d'un spectromètre (FT-IR, Modèle Bio-rad FTS-40).

Détermination du caractère acido-basique et pH $_{Z P C}$ du charbon actif

Le caractère acido-basique $(\mathrm{pH}$ de contact) d'un charbon actif est déterminé par la mesure du $\mathrm{pH}$ de contact. A cet effet, 100 $\mathrm{mg}$ de charbon actif sont placés dans $50 \mathrm{~mL}$ d'une solution de $\mathrm{NaCl}$ 0,01 mol.L $\mathrm{L}^{-1}$ contenus dans un adsorbeur (LACOP ADS X-2). La suspension est laissée sous agitation pendant 72 heures, et ensuite centrifugée. Le $\mathrm{pH}$ du centrifugeât obtenu correspond au $\mathrm{pH}$ de contact, qui détermine la nature acide ou basique du charbon actif. Le $\mathrm{pH}_{\mathrm{ZPC}}$ a été déterminé par la méthode de dérive de $\mathrm{pH}$, en utilisant $100 \mathrm{mg}$ de charbon actif et $50 \mathrm{~mL}$ des solutions de $\mathrm{NaCl} 0,01 \mathrm{M}$ de $\mathrm{pH}$ variant de 2 à 12 (Edwin Vasu, 2008), pH ajusté par addition d'une solution de $\mathrm{HCl} 0,01 \mathrm{~N}$ ou $\mathrm{KOH} 0,01$ M.

Détermination des paramètres de conformité aux normes de la pharmacopée

Dans l'optique d'une utilisation médicale du charbon actif préparé, nous avons évalué ses propriétés au regard des exigences 
de conformité aux normes de la pharmacopée. la pharmacopée ont été déterminés selon les méthodes classiques décrites par l'USpharmacopeial (1985). Les tests ci-après ont été effectués: la limite microbienne, les chlorures, les sulfates, les composés non carbonisés, les sulfures, la perte à la dessiccation, les substances solubles dans les acides, les composés cyanogénés, les résidus de calcination, les métaux lourds et le pouvoir décolorant.

\section{Essais d'adsorption}

Les essais d'adsorption ont été effectués en réacteur discontinu à $28 \pm 1{ }^{\circ} \mathrm{C}$ avec des solutions aqueuses de $\mathrm{QBC}$ au $\mathrm{pH}$ d'auto-équilibre $(5,82)$ ou fixé ( $\mathrm{pH} 1,05,2,00$, 2,91) à l'aide d'une solution commerciale de HC $\ell$ (MERCK, 37\%, d 1,19), avant l'addition de l'antipaludéen. Le $\mathrm{pH}$ des solutions, avant et après adsorption, est mesuré avec un pHmètre (HANNA-Instruments). La Figure 1 donne la structure de la quinine.

Nous avons travaillé dans des conditions micromolaires pour réduire les interactions parallèles entre molécules d'adsorbat et favoriser les interactions verticales entre adsorbat et adsorbant. A cet effet, $50 \mathrm{~mL}$ de la solution de QBC $28.10^{-6} \mathrm{M}$, placés dans un adsorbeur hermétique de 500 mL (LACOP ADS X-2), sont mélangés avec 1 mg de CAP. La suspension est agitée au temps (t) requis pour l'essai. Le temps d'agitation est varié de 0 à 90 minutes. Après agitation, la
Les paramètres de conformité aux normes de suspension est centrifugée à 3000 r.p.m (Labofuge 200 Heraeus) pendant 30 minutes. Le centrifugeât est analysé au spectrophotomètre UV-Vis (BECKMAN DU 250 ; General purpose) à la longueur d'onde $\left(\lambda_{\max }\right)$ de $251 \mathrm{~nm}$ pour les $\mathrm{pH} 1,05,2,00,2,91$ et $235 \mathrm{~nm}$ pour le $\mathrm{pH} 5,83$, pour en déterminer la concentration à l'équilibre. Des essais témoins effectués sans charbon actif ont permis de s'assurer qu'il n'y a aucune perte du composé organique au cours de l'agitation ou de la centrifugation de la suspension (Composé organique- Charbon actif).

La capacité d'adsorption $\mathrm{Q}_{\mathrm{e}}\left(\mathrm{mg} . \mathrm{g}^{-1}\right)$ du CAP est calculée par la relation ci-dessous :

$\mathrm{Q}_{\mathrm{e}}=\frac{\left(\mathrm{C}_{\mathrm{o}}-\mathrm{C}_{\mathrm{e}}\right) \cdot \mathrm{M}_{\mathrm{QBC}} \cdot \mathrm{V}}{\mathrm{m}_{\mathrm{C}}}$

Avec: $Q_{e}$, la capacité d'adsorption à l'équilibre (mg. $\left.\mathrm{g}^{-1}\right) ; \mathrm{C}_{0}$, la concentration initiale de la solution de QBC $\left(\operatorname{mol} . \mathrm{L}^{-1}\right) ; \mathrm{C}_{\mathrm{e}}$, la concentration à l'équilibre de la solution de QBC $\left(\operatorname{mol} . \mathrm{L}^{-1}\right) ; \mathrm{M}_{\mathrm{QBC}}$, la masse molaire de QBC $\left(\mathrm{g} \cdot \mathrm{mo} \ell^{-1}\right) ; \mathrm{m}_{\mathrm{C}}$, la masse du charbon actif $(\mathrm{g}) ; \mathrm{V}$, le volume de la solution $(\mathrm{mL})$. Le pourcentage d'adsorption (\%), qui donne le rapport du nombre des moles du soluté adsorbées par rapport au nombre de moles initiales, est calculé d'après la relation :

$$
\%=\frac{\mathrm{C}_{\mathrm{o}}-\mathrm{C}_{\mathrm{e}}}{\mathrm{C}_{\mathrm{o}}} \times 100
$$

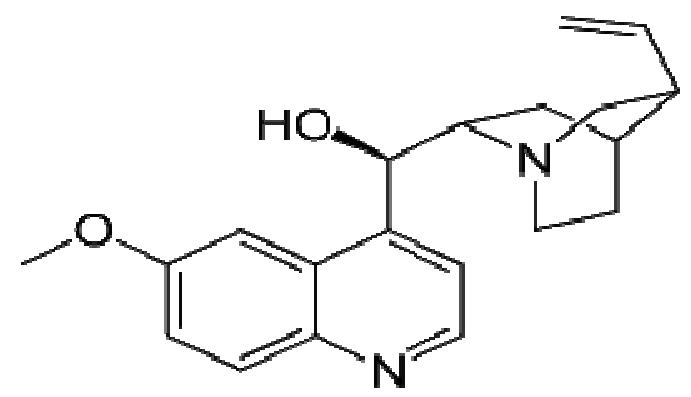

Figure 1 : Structure de la quinine.

\section{Détermination des isothermes d'adsorption}


Les isothermes d'adsorption ont été obtenues en effectuant les essais d'adsorption en fonction de la concentration de la Quinine Bichlorhydrate. $50 \mathrm{~mL}$ des solutions de QBC de concentration $10^{-6} \mathrm{~mol} . \mathrm{L}^{-1}$ à $28.10^{-6}$ mol. $\mathrm{L}^{-1}$ ont été utilisés, pour les différents $\mathrm{pH}$. La masse du charbon actif utilisée est de 1 $\mathrm{mg}$. Le temps d'agitation pour l'adsorption est fixé à 60 minutes, temps se situant dans le domaine de l'équilibre pour tous les systèmes CAP-QBC étudiés.

\section{RESULTATS}

Tous les résultats numériques sont exprimés avec un intervalle de confiance égal ou supérieur à $96 \%$.

\section{Rendement de préparation et pouvoir adsorbant du charbon actif}

Les résultats obtenus montrent que les sciures non imprégnées donnent le meilleur rendement $(30 \%)$ comparé à celui obtenu avec les sciures imprégnées (23\%). Maria et al. (2010) ont obtenu un rendement en charbon actif de $48,16 \%$ à partir d'autres matériaux précurseurs. La diminution de rendement observée en présence d'un agent d'imprégnation est due probablement à la réaction de l'agent d'imprégnation avec des substances carbonées (cellulose, hemicelluloses, lignine, etc.) contenues dans les sciures, solubilisant ainsi une quantité importante des matières carbonées.

L'activité du charbon actif est exprimée par son pouvoir adsorbant qui correspond à la capacité d'adsorption à saturation du charbon actif. Elle est généralement déterminée par adsorption du bleu de méthylène. Le Tableau 1 donne le pouvoir adsorbant des charbons préparés et de deux charbons commerciaux étudiés dans les mêmes conditions que nos charbons.

En comparant l'activité du charbon des sciures non imprégnées à celle du charbon des sciures imprégnées dans l'acide phosphorique, on constate que le PA de ce dernier est plus élevé de 73 fois pour l'adsorption de BM et à deux fois pour l'adsorption de l'iode. Le charbon actif préparé avec l'acide phosphorique a une activité plus grande par rapport aux charbons commerciaux MERCK (CM) et CECA (Tableau 1). L'activation thermique à $900{ }^{\circ} \mathrm{C}$ facilite le développement de la microporosité ouverte, c'est-à-dire dont les pores sont ouverts à la surface. On assiste ainsi à une dépolymérisation du matériau carboné (précurseur) aux basses températures et à partir de $400{ }^{\circ} \mathrm{C}$, il y a une réorganisation de la structure du matériau qui conduit à l'aromatisation de la structure. Il y a formation des couches polyaromatiques (graphènes) déposées en plans basaux à forme graphitique (Mangun et al., 1999).

\section{Propriétés physiques du charbon actif BP}

L'image SEM du charbon actif BP est donnée par la Figure 2. L'observation au microscope électronique à balayage montre clairement une porosité très développée sur toute la surface. On observe une certaine hétérogénéité structurale des pores.

Le charbon actif BP présente une densité de micropores très élevée. Le diamètre moyen des pores est évalué à $50 \mathrm{~nm}$; il caractérise les charbons mesoporeux (diamètre moyen des pores, situé entre 2 et 50 $\mathrm{nm})$. La surface spécifique du charbon actif BP est $1439,46 \mathrm{~m}^{2} / \mathrm{g}$, surface suffisamment grande qui peut justifier une forte adsorption des molécules organiques sur ce charbon. La surface spécifique déterminée est de loin supérieure à celle du charbon DARCO (produit Aldrich) $\left(550 \mathrm{~m}^{2} / \mathrm{g}\right)$ ou d'un charbon actif en poudre $\left(658 \mathrm{~m}^{2} / \mathrm{g}\right)$ utilisé par Bouchemal et Achour (2007). Elle est par contre inférieure à celle $\left(1688 \mathrm{~m}^{2} / \mathrm{g}\right) \mathrm{du}$ charbon actif à base d'Enteromorpha prolifera, préparé par Li et al. (2010). 
Nature du charbon actif, $\mathbf{p H}_{\mathrm{ZPC}}$, composition élémentaire et contenu en cendres

Le charbon actif BP est acide (pHcontact 6,2); son $\mathrm{pH}_{\mathrm{ZPC}}$ est 6,2. En dessous du pH 6,2, le charbon BP est chargé positivement, tandis qu'au-delà de ce $\mathrm{pH}$, sa surface est chargée négativement.

Le spectre EDS du charbon actif BP est donné dans la Figure 3. La composition élémentaire se présente de la manière suivante : $93,57 \% \mathrm{C}, 6,32 \% \mathrm{O}$ et $0,11 \% \mathrm{P}$. Le spectre EDS présente un pic à $2,00 \mathrm{keV}$, qui a été attribué au phosphore. Ce phosphore résiduel provient uniquement de l'agent d'imprégnation $\left(\mathrm{H}_{3} \mathrm{PO}_{4}\right)$ utilisé. L'azote ou le soufre ne sont pas présents. La composition du charbon actif BP se trouve dans le même ordre que celles rapportées dans la littérature et avec un pourcentage en carbone nettement plus élevé, dans la plupart des cas (Li, 2002; Balci et al., 1994).

Les Figures 4 et 5 sont une représentation des images TEM et XRD pour le charbon actif $\mathrm{BP}$, respectivement. L'absence des anneaux de diffraction dans l'image TEM et celle des pics dans la gamme de l'angle $2 \theta$ étudiée (Figures 3 et 4 ) indiquent l'absence de cristallisation dans ce charbon. Le charbon préparé est donc du carbone amorphe et poreux, donc un charbon actif.

Le contenu en cendres du charbon actif préparé est très faible $(1,6 \%)$ et bien loin de la valeur maximale tolérable pour le charbon actif (15\%) (US XXI,1985). Cette valeur est inférieure à celles des pyrolysats de la Bagasse de canne à sucre et du Vétiver obtenues par Osnick (2009).

\section{Fonctions chimiques de surface du charbon} actif

Analyse par la méthode de Boehm

Nous avons identifié les fonctions de surface de type acide, et les fonctions de surface de type basique sur le charbon actif BP et le charbon actif MERCK, utilisé comme référence. Les résultats obtenus montrent, pour tous les charbons actifs, la présence des fonctions acides de surface de type carboxylique $(-\mathrm{COOH})$, lactone $(-\mathrm{COO})$, phénol $(-\mathrm{OH})$, et les fonctions basiques de surface de type chromène ou pyrone (Tableau 2).

Pour chaque charbon actif, les fonctions de surface de type acide sont de loin plus prépondérantes comparées aux fonctions de surface basiques : 31,75 fois plus pour le CAP BP et 13,13 fois plus pour le charbon actif CM. Les fonctions carboxyliques sont prépondérantes par rapport aux autres fonctions acides.

\section{Analyse par FT-IR}

L'évaluation complète des fonctions chimiques de surface a été faite par FT-IR. Le spectre obtenu est présenté dans la Figure 6. Le Tableau 3 donne les fonctions chimiques identifiées.

\section{Paramètres de conformité aux normes de la pharmacopée}

L'utilisation du charbon actif préparé dans la désintoxication nécessite que ce charbon actif réponde aux exigences de conformité aux normes de la pharmacopée. Les résultats de la pharmacopée obtenus indiquent que le charbon actif $\mathrm{BP}$ et le charbon $\mathrm{CM}$ ne contiennent pas des germes bactériens indicateurs de contamination biologique (Tableau 4). Tous les charbons actifs sont exempts des substances indésirables, notamment les chlorures, sulfates, sulfures, composés cyanogénés, composés non carbonisés et les métaux lourds.

\section{Adsorption de QBC sur CAP BP \\ Etude de la cinétique d'adsorption \\ Les courbes cinétiques pour l'adsorption de QBC sur CAP BP sont}


données par la Figure 7 et montrent que la capacité d'adsorption augmente avec le temps d'adsorption jusqu'à atteindre une valeur maximale $\left(Q_{m}\right)$ qui ne change plus malgré l'augmentation $\mathrm{du}$ temps. Les courbes cinétiques obtenues sont des courbes typiques de saturation. Les sites d'adsorption sont occupés progressivement par les molécules de QBC jusqu'à leur saturation. Le temps d'équilibre est atteint à 40 minutes pour tous les $\mathrm{pH}$. La capacité d'adsorption augmente avec le $\mathrm{pH}$ de la solution (Tableau 5). En milieu acide $\left(\mathrm{pH}<\mathrm{pH} \mathrm{ZPC}_{\mathrm{ZPC}}\right)$, il y a coadsorption de QBC et des protons sur la surface du charbon actif. Les fonctions oxygénées de surface du charbon sont protonées, ce qui entraîne des interactions répulsives avec les ions cationiques organiques de QBC, d'où la diminution de la capacité d'adsorption du charbon actif en milieu fortement acide.

Les valeurs d'enthalpies ont été déterminées en utilisant la relation de Clausius- Clapeyron (Avom et al, 2001):

$$
\operatorname{Ln} \mathrm{C}^{\infty}=\frac{\Delta \mathrm{H}_{\mathrm{ads}}}{\mathrm{R} \mathrm{T}}
$$

Où, $\mathrm{C}^{\infty}$, est la concentration du soluté au cœur de la solution, correspondant à $\mathrm{Q}_{\mathrm{m}}\left(\mathrm{mol} . \mathrm{L}^{-1}\right) ; \quad \Delta \mathrm{H}_{\mathrm{ads}}, \quad$ la variation de l'enthalpie d'adsorption, correspondant à la chaleur d'adsorption, $\mathrm{Q}_{\text {ads }}\left(\mathrm{kJ} \cdot \mathrm{mol}^{-1}\right) ; \mathrm{T}$, la température d'adsorption $(\mathrm{K})$; R, la constante molaire des gaz parfaits $\left(8,31 \mathrm{~J} \cdot \mathrm{mo}^{-1} \cdot \mathrm{K}^{-1}\right)$. Les valeurs obtenues indiquent que l'adsorption de QBC sur le CAP BP est exothermique. Ces valeurs d'enthalpie d'adsorption inférieures à $40 \quad \mathrm{~kJ} \cdot \mathrm{mol}^{-1}$ suggèrent que l'adsorption de QBC sur le charbon actif BP est essentiellement physique, et, gouvernée principalement par les interactions de Van der Waals et les interactions de type électrostatique. Hafiz et al. (2011) évoquent aussi des attractions électrostatiques pour justifier l'adsorption des composés organiques volatils (COVs) sur charbon actif en grains.

\section{Modélisation de la cinétique d'adsorption}

La modélisation de la cinétique d'adsorption a été faite à l'aide des modèles cinétiques de réaction de surface de pseudoordre 1 et 2 , du modèle cinétique d'Elovich et $\mathrm{du}$ modèle cinétique de diffusion intraparticulaire.

\section{Modèles cinétiques de réaction de surface de pseudo-ordre 1 et pseudo-ordre 2}

Les modèles cinétiques couramment utilisés pour la réaction de surface sont ceux de pseudo-ordre 1 et de pseudo-ordre 2. Les équations habituellement utilisées sont les suivantes :

Modèle de pseudo-ordre 1 ou modèle de Lagergren (Ho et McKay, 1998 ; Srivastava et al., 2006) :

$$
\begin{aligned}
& \frac{\mathrm{d}_{\mathrm{t}}}{\mathrm{dt}}=\mathrm{k}_{1}\left(\mathrm{q}_{\mathrm{e}}-\mathrm{q}_{\mathrm{t}}\right) \\
& \ell \mathrm{n}\left(\mathrm{q}_{\mathrm{e}}-\mathrm{q}_{\mathrm{t}}\right)=\ell \mathrm{n} \mathrm{q}_{\mathrm{e}}-\mathrm{k}_{1} \mathrm{t}
\end{aligned}
$$

Avec : $\mathrm{q}_{\mathrm{t}}$, la capacité d'adsorption au temps $\mathrm{t}$; $\mathrm{q}_{\mathrm{e}}$, la capacité d'adsorption à l'équilibre et $\mathrm{k}_{1}$, la constante de vitesse de pseudo-ordre 1.

Modèle de pseudo-ordre 2, dont il existe cinq développements classiques (Ho et Mc Kay, 2000 ; Kumar, 2007).

$$
\frac{\mathrm{d} \mathrm{q}_{\mathrm{t}}}{\mathrm{dt}}=\mathrm{k}_{2}\left(\mathrm{q}_{\mathrm{e}}-\mathrm{q}_{\mathrm{t}}\right)^{2}
$$

Avec $\mathrm{k}_{2}$, la constante de vitesse de pseudoordre 2 .

Dans cette étude, nous avons développé une nouvelle approche mathématique pour la réaction de surface de pseudo-ordre 1 et pseudo- ordre 2.

L'équilibre d'adsorption d'un soluté A sur le charbon actif peut être représenté par l'équation ci-après :
adsorption
$(S)_{s}+A$
désorption
$(S A)_{S}$ 
Au temps $\mathrm{t}=0, \quad \mathrm{q}_{\mathrm{e}}\left(\right.$ ou $\left.\mathrm{q}_{\mathrm{m}}\right) \quad \mathrm{C}_{\mathrm{o}}$ 0

Au temps $\mathrm{t}=\mathrm{t}, \quad\left(\right.$ ou $\left.\mathrm{q}_{\mathrm{m}}-\mathrm{q}_{\mathrm{t}}\right) \mathrm{C}_{\mathrm{t}}$ ou $\mathrm{C}_{\mathrm{e}} \mathrm{q}_{\mathrm{t}}$

Au temps $\mathrm{t}=0$, la concentration des sites libres sur le solide $(\mathrm{S})_{\mathrm{s}}$ est $\mathrm{q}_{\mathrm{e}}\left(\right.$ ou $\mathrm{q}_{\mathrm{m}}$ ); la concentration du soluté en solution est $\mathrm{C}_{\mathrm{o}}$, et, celle du soluté fixé sur les sites libres $(\mathrm{SA})_{\mathrm{s}}, 0$. Au temps $\mathrm{t}=\mathrm{t}$, la concentration du soluté fixé sur le solide est $\mathrm{q}_{\mathrm{t}}$, celle des sites libres devient $\mathrm{q}_{\mathrm{e}}-\mathrm{q}_{\mathrm{t}}$ (ou $\mathrm{q}_{\mathrm{m}}-\mathrm{q}_{\mathrm{t}}$ ) et celle du soluté $\mathrm{au}$ cœur de la solution $\mathrm{C}_{\mathrm{t}}\left(\right.$ ou $\left.\mathrm{C}_{\mathrm{e}}\right)$. Au temps $\mathrm{t}=0$, la concentration du soluté $\mathrm{A}$ en solution est assimilable à $\mathrm{q}_{\mathrm{e}}$, la concentration de $\mathrm{A}$ adsorbée quand l'équilibre est atteint. $\mathrm{Au}$ temps $\mathrm{t}=\mathrm{t}$, la concentration du soluté $\mathrm{A}$, libre en solution est assimilable à $\mathrm{q}_{\mathrm{e}}-\mathrm{q}_{\mathrm{t}}$, la concentration des sites libres du solide.

Nouvelle approche de la réaction de surface de pseudo-ordre 1

L'équilibre ci-dessus peut-être simplifié de la manière suivante :

$$
\mathrm{A} \rightleftharpoons \mathrm{A}_{(\mathrm{s})}
$$

$\begin{array}{lcl}A \mathrm{t}=0 & \mathrm{q}_{\mathrm{e}} & 0 \\ \mathrm{~A} \mathrm{t}=\mathrm{t} & \mathrm{q}_{\mathrm{e}}-\mathrm{q}_{\mathrm{t}} & \mathrm{q}_{\mathrm{t}}\end{array}$

Avec A, le soluté au cœur de la solution et $\mathrm{A}(\mathrm{s})$ le soluté fixé sur le charbon actif. La vitesse de réaction de cette réaction est définie par

$$
\mathrm{v}=\mathrm{k}_{1}[\mathrm{~A}]=-\frac{\mathrm{d}[\mathrm{A}]}{\mathrm{d} \mathrm{t}}
$$

En séparant de part et d'autre du signe égal chacune des deux variables indépendantes, et, en intégrant l'équation obtenue dans les limites de $[\mathrm{A}]_{0}$ à $[\mathrm{A}]_{\mathrm{t}}$ et de $\mathrm{t}=0$ à $\mathrm{t}=\mathrm{t}$, on a :

$$
\ln [\mathrm{A}]_{\mathrm{t}}=\ln [\mathrm{A}]_{\mathrm{o}}-\mathrm{k}_{1} \mathrm{t}
$$

En remplaçant $[\mathrm{A}]_{0}$ et $[\mathrm{A}]_{t}$ par $\mathrm{q}_{\mathrm{e}}$ et $\mathrm{q}_{\mathrm{e}}$ $\mathrm{q}_{\mathrm{t}}$, respectivement, on trouve l'équation empirique de Lagergren:

$$
\ell \mathrm{n}\left(\mathrm{q}_{\mathrm{e}}-\mathrm{q}_{\mathrm{t}}\right)=\ell \mathrm{n} \mathrm{q}_{\mathrm{e}}-\mathrm{k}_{1} \mathrm{t}
$$

Le développement de l'équation (12) conduit à une nouvelle équation, non encore utilisée en cinétique hétérogène:

$\ln \frac{\mathrm{q}_{\mathrm{e}}}{\left(\mathrm{q}_{\mathrm{e}}-\mathrm{q}_{\mathrm{t}}\right)}=\mathrm{k}_{1} \mathrm{t}$

En portant $\ln \frac{\mathrm{q}_{\mathrm{e}}}{\left(\mathrm{q}_{\mathrm{e}}-\mathrm{q}_{\mathrm{t}}\right)}$ en fonction $\mathrm{du}$ temps, $t$, on obtient une droite dont la pente correspond à $\mathrm{k}_{1}$, la constante de vitesse de la réaction d'adsorption de surface de pseudoordre 1. On peut définir le temps de demiadsorption comme le temps nécessaire au bout duquel la moitié de la concentration du soluté adsorbable est adsorbée.

Donc :

$$
\mathrm{t}_{1 / 2}=\frac{0,693}{\mathrm{k}_{1}}
$$

Avec $: \mathrm{t}_{1 / 2}$, le temps de demi-adsorption (s) $; \mathrm{k}_{1}$, la constante de vitesse de la réaction d'adsorption de surface de pseudo-ordre 1 . Lorsque l'ordonnée à l'origine de cette droite expérimentale tend vers zéro, cela indique que le système étudié est mieux décrit avec la cinétique de pseudo-ordre1; ou bien un coefficient de corrélation proche de l'unité.

Nouvelle approche de la réaction de surface de pseudo-ordre 2.

Soit une réaction élémentaire

$$
\mathrm{A}+\mathrm{B} \rightarrow \mathrm{P}
$$

Si $[\mathrm{A}]_{0}$ est différente de $[\mathrm{B}]_{0}$, la vitesse de cette réaction est donnée par :

$$
\mathrm{v}=-\frac{\mathrm{d}[\mathrm{A}]}{\mathrm{dt}}=-\frac{\mathrm{d}[\mathrm{B}]}{\mathrm{d} \mathrm{t}}=\mathrm{k}_{2}[\mathrm{~A}][\mathrm{B}](16)
$$

On peut aussi écrire :

$$
\mathrm{v}=\frac{\mathrm{d} \mathrm{x}}{\mathrm{dt}}=\mathrm{k}_{2}(\mathrm{a}-\mathrm{x})(\mathrm{b}-\mathrm{x})
$$

Avec: v, la vitesse de la réaction; a, la concentration du réactif $\mathrm{A}$ au temps initial, $\mathrm{t}=$ $0 ; \mathrm{b}$, la concentration du réactif $\mathrm{B}$ au temps initial, $\mathrm{t}=0 ; \mathrm{x}$, la concentration du réactif $\mathrm{A}$ ou $\mathrm{B}$, ayant réagi au temps $\mathrm{t}$; $\mathrm{a}-\mathrm{x}$, la 
concentration du réactif $\mathrm{A}$, au temps $\mathrm{t} ; \mathrm{b}-\mathrm{x}$, la concentration du réactif $B$, au temps $t$. Dans le cas où $\mathrm{a}=\mathrm{b}$, l'équation de vitesse (17) devient :

$$
\frac{\mathrm{d} x}{\mathrm{~d} \mathrm{t}}=\mathrm{k}_{2}(\mathrm{a}-\mathrm{x})^{2}
$$

Dans le cas de l'adsorption, et, en posant comme pour l'équation de vitesse de réaction d'adsorption de surface de pseudoordre $1, \quad a=q_{e}$ et $x=q_{t}$, on retrouve l'équation empirique de vitesse de la réaction d'adsorption de pseudo-ordre 2.

$$
\frac{\mathrm{d} \mathrm{q}_{\mathrm{t}}}{\mathrm{dt}}=\mathrm{k}_{2}\left(\mathrm{q}_{\mathrm{e}}-\mathrm{q}_{\mathrm{t}}\right)^{2}
$$

Après séparation des variables indépendantes, et intégration de l'équation obtenue dans les conditions limites $\left(\mathrm{t}=0\right.$ à $\mathrm{t}=\mathrm{t} ; \mathrm{q}_{\mathrm{t}}=0$ à $\mathrm{q}_{\mathrm{t}}=\mathrm{q}_{\mathrm{e}}$ ), on a une nouvelle équation non encore utilisée en cinétique hétérogène :

$$
\frac{\mathrm{q}_{\mathrm{t}}}{\mathrm{q}_{\mathrm{e}}\left(\mathrm{q}_{\mathrm{e}}-\mathrm{q}_{\mathrm{t}}\right)}=\mathrm{k}_{2} \mathrm{t}
$$

Lorsqu'on porte $\frac{\mathrm{q}_{\mathrm{t}}}{\mathrm{q}_{\mathrm{e}}\left(\mathrm{q}_{\mathrm{e}}-\mathrm{q}_{\mathrm{t}}\right)}$ en fonction de $t$, on obtient une droite dont la pente correspond à $\mathrm{k}_{2}$, la constante de vitesse de la réaction d'adsorption de surface de pseudoordre 2. Le temps de demi-adsorption pour la réaction d'adsorption de pseudo-ordre 2 est donné par l'équation ci-après :

$$
\mathrm{t}_{1 / 2}=\frac{1}{\mathrm{k}_{2} \cdot \mathrm{q}_{\mathrm{e}}}
$$

En pratique, un coefficient de corrélation $\mathrm{R}^{2}$ proche de 1 obtenu, en portant $\frac{\mathrm{q}_{\mathrm{t}}}{\mathrm{q}_{\mathrm{e}}\left(\mathrm{q}_{\mathrm{e}}-\mathrm{q}_{\mathrm{t}}\right)}$ en fonction de $\mathrm{t}$ indique que la réaction de surface est de pseudo-ordre 2 . L'ordonnée à l'origine tend à zéro dans ce cas. Comme pour la réaction d'adsorption de pseudo-ordre 1 , on peut aussi déterminer $t_{1 / 2}$ expérimentalement en portant $\mathrm{q}_{\mathrm{e}}$ en fonction du temps t.

\section{Modèle d'Elovich}

Le modèle d'Elovich a été utilisé sous sa forme simplifiée (Chien et Clayton, 1980):

$$
\mathrm{q}_{\mathrm{t}}=\frac{1}{\beta} \ln (\alpha \beta)+\frac{1}{\beta} \ln (t)
$$

Où : $\mathrm{q}_{\mathrm{t}}$, la concentration du soluté adsorbé au temps $\mathrm{t}\left(\mathrm{mg} \cdot \mathrm{g}^{-1}\right) ; \alpha$, la constante de vitesse initiale $\left(\mathrm{mg} \cdot \mathrm{g}^{-1} \cdot \mathrm{min}^{-1}\right) ; \beta$, la constante d'adsorption $\left(\mathrm{g} . \mathrm{mg}^{-1}\right)$ au cours de n'importe quelle expérience; $\mathrm{t}$, le temps d'adsorption. En portant $\mathrm{q}_{\mathrm{t}}$ en fonction de lnt, on obtient une droite dont la pente et l'ordonnée à l'origine permettent la détermination de $\beta$ et $\alpha$, respectivement.

\section{Modèle de diffusion intraparticulaire}

Le modèle de diffusion intraparticulaire de Weber et Morris simplifié suivant a été utilisé (Srivastava et al., 2006)

$$
\log \mathrm{R}=\log \mathrm{K}_{\mathrm{di}}+\mathrm{alog} \mathrm{t}
$$

Où : $\mathrm{R}$, le pourcentage de soluté adsorbé ; $\mathrm{t}$, le temps d'adsorption ( $\mathrm{min}) ; \mathrm{k}_{\mathrm{id}}$, la constante de vitesse de diffusion intraparticulaire (mg.g. $\left.{ }^{1} \min ^{-1}\right)$; a , une constante en relation avec le mécanisme d'adsorption.

Les droites obtenues pour les modèles cinétiques de pseudo-ordre 1 et 2 , le modèle d'Elovich et le modèle de diffusion intraparticulaire sont consignées dans les Figures 8 à 11. Les paramètres correspondant à chaque modèle sont repris dans les Tableaux 6 à 8.

A la lumière de ces résultats, il ressort que les modèles d'adsorption de la réaction de surface de pseudo-ordre 1 et pseudo-ordre 2, d'Elovich et de diffusion intraparticulaire sont suivis par le système (QBC-BP) aux quatre $\mathrm{pH}$ étudiés (coefficients de corrélation supérieurs à 0,83 dans tous les cas). Les constantes de vitesse de pseudo- ordre 1 sont plus grandes, comparées aux constantes de vitesse de pseudo-ordre 2, ce qui suppose que la fixation des molécules de QBC sur les CAP s'effectue plus rapidement en début 
d'adsorption sur les premiers types des sites d'adsorption et, avec le temps et plus lentement, les molécules du soluté se fixent sur les deuxièmes types de sites. D'une manière générale, on observe une influence du $\mathrm{pH}$ sur les constantes de vitesse $\mathrm{k}_{1}$ et $\mathrm{k}_{2}$ et sur les autres paramètres cinétiques des modèles étudiés.

Etude des isothermes d'adsorption de QBC sur CAP BP

Les isothermes d'adsorption de QBC sur CAP BP sont données par la Figure 12. La forme de ces isothermes montre qu'elles sont dans la majorité de type $\mathrm{S}$ pour l'adorption du QBC aux pH 1,05; 2,91; et 5, 82. Seule l'isotherme obtenue à $\mathrm{pH} 2,00$ est de type L. La forme $\mathrm{S}$ des isothermes d'adsorption de QBC indique une adsorption multicouches de QBC sur ce charbon actif. Les concavités des isothermes S sont tournées vers le haut aux faibles concentrations pour l'adsorption de $\mathrm{QBC}$ à $\mathrm{pH} 1,05$ et 5,82. Cette concavité indique la formation des multicouches dès les faibles concentrations du soluté et l'existence des interactions fortes entre les molécules du soluté (QBC) comparées aux interactions entre molécules du soluté et adsorbant (BP). Ce qui traduit une forte affinité entre $\mathrm{QBC}$ et $\mathrm{BP}$ au $\mathrm{pH}$ très acide $(1,05)$, et au $\mathrm{pH}$ faiblement acide $(5,82)$, en absence de l'acide chlorhydrique. L'isotherme $\mathrm{S}$ obtenue à $\mathrm{pH}$ 2,91 a une concavité tournée vers le bas aux faibles concentrations et traduit une adsorption coopérative. Les molécules adsorbées favorisent la fixation d'autres molécules encore libres à la surface interne du charbon actif. Il y a en ce moment la formation de plusieurs couches supplémentaires. Il y a donc une forte compétition d'adsorption entre le soluté et le solvant pour les sites d'adsorption du charbon actif. Le point d'inflexion qui apparaît pour les courbes $\mathrm{S}$ traduit l'existence d'au moins deux mécanismes d'adsorption : d'abord une adsorption de QBC sur les surfaces ouvertes du CAP et ensuite la formation de nouvelles couches d'adsorption dues aux interactions entre les molécules adsorbées du soluté et celles non adsorbées. Cette forme d'isotherme $\mathrm{S}$ avec concavité tournée vers le bas aux faibles concentrations indique aussi que les molécules du soluté (QBC) s'adsorbent verticalement sur les micropores du charbon actif.

\section{Isotherme de Langmuir}

La modélisation des isothermes d'adsorption est faite en recourant aux isothermes de Langmuir et de Freundlich. L'isotherme de Langmuir en phase liquide est donnée par (Edwin Vasu, 2008 ; Matos et al., 2011):

$$
\frac{\mathrm{Q}_{\mathrm{e}}}{\mathrm{Q}_{\mathrm{m}}}=\theta=\frac{\mathrm{K}_{\mathrm{L}} \mathrm{Ce}}{1+\mathrm{K}_{\mathrm{L}} \mathrm{Ce}}
$$

Cette équation a été traitée sous sa forme linéarisée, dite Langmuir 1 :

$$
\frac{1}{\mathrm{Q}_{\mathrm{e}}}=\frac{1}{\mathrm{Q}_{\mathrm{m}}}+\frac{1}{\mathrm{Q}_{\mathrm{m}} \cdot \mathrm{K}_{\mathrm{L}}} \cdot \frac{1}{\mathrm{C}_{\mathrm{e}}}
$$

Avec: $\mathrm{Q}_{\mathrm{e}}$, la capacité d'adsorption du CAP à l'équilibre $\left(\mathrm{mg} \cdot \mathrm{g}^{-1}\right) ; \mathrm{Q}_{\mathrm{m}}$, la capacité maximale d'adsorption du CAP (mg.g $\left.{ }^{-1}\right) ; \quad \mathrm{K}_{\mathrm{L}}$, la constante d'équilibre d'adsorption (constante de Langmuir) (L.mo $\left.\ell^{-1}\right) ; \mathrm{C}_{\mathrm{e}}$, la concentration du soluté à l'équilibre $\left(\mathrm{mo} \ell \cdot \mathrm{L}^{-1}\right)$. En portant $\frac{1}{Q_{e}}$ en fonction $1 / C_{e}$, l'équation de Langmuir linéarisée permet la détermination de deux paramètres importants de l'adsorption, à savoir la capacité ultime d'adsorption $\left(\mathrm{Q}_{\mathrm{m}}\right)$ et la constante d'équilibre d'adsorption $\left(\mathrm{K}_{\mathrm{L}}\right)$, à partir de l'ordonnée à l'origine et de la pente, respectivement. Le paramètre d'équilibre, adimensionnel, $\mathrm{R}_{\mathrm{L}}$, a aussi été calculé à l'aide de la relation suivante (Karthikejan et al., 2005):

$\mathrm{R}_{\mathrm{L}}=\frac{1}{1+\mathrm{K}_{\mathrm{L}} \mathrm{C}_{\mathrm{o}}}$

Avec: $\mathrm{R}_{\mathrm{L}}$, le paramètre d'équilibre; $\mathrm{K}_{\mathrm{L}}$, la constante d'adsorption $\left(\mathrm{L}^{\mathrm{m}} \mathrm{mol}^{-1}\right) ; \mathrm{C}_{\mathrm{o}}$, la 
concentration initiale de la solution de QBC $\left(\right.$ mol.L $\left.L^{-1}\right)$ Les valeurs calculées des paramètres $\mathrm{Q}_{\mathrm{m}}$ et $\mathrm{K}_{\mathrm{L}}$ du modèle de Langmuir ainsi que les coefficients de corrélation sont présentés dans le Tableau 9.

Les résultats du Tableau 9 montrent que $\mathrm{Q}_{\mathrm{m}}$ diminue faiblement lorsqu'on passe du $\mathrm{pH}$ 1,05 au pH 5,82 alors que $\mathrm{K}_{\mathrm{L}}$ augmente suffisamment. Le coefficient de corrélation global $\left(\mathrm{R}^{2} \mathrm{~g}\right)$ indique que l'isotherme de Langmuir est suivie par le système QBC - BP, surtout à $\mathrm{pH} 1,05$ et 2,00. Le $\mathrm{pH}$ influence suffisamment l'affinité de QBC sur le charbon actif BP. Un accroissement du $\mathrm{pH}$ de la solution de 1,05 à 5,82 entraîne l'accroissement de l'affinité de QBC pour le CAP BP. Les résultats obtenus pour le paramètre $\mathrm{K}_{\mathrm{L}}$ indiquent une affinité élevée et une forte énergie d'adsorption pour le soluté QBC. L'affinité la plus forte $\quad\left(1,902.10^{5}\right.$ L.mol ${ }^{-1}$ ) est obtenue à pH 5,82. L'affinité de QBC pour le CAP BP s'explique par la structure de surface du CAP.

\section{Isotherme de Freundlich}

L'isotherme de Freundlich est donnée par la relation (Srivastava, 2006) :

$\mathrm{Q}_{\mathrm{e}}=\mathrm{K}_{\mathrm{F}} \cdot \mathrm{C}_{\mathrm{e}}^{1 / \mathrm{n}}$.
Nous avons utilisé l'équation de Freundlich sous sa forme linéaire :

$\log Q_{e}=\log K_{F}+1 / n \log C_{e}$

Avec : $\mathrm{Q}_{\mathrm{e}}$, la capacité d'adsorption du CAP à l'équilibre $\left(\mathrm{mg} \cdot \mathrm{g}^{-1}\right) ; \mathrm{K}_{\mathrm{F}}$, la constante de Freundlich $\left((\mathrm{mg} / \mathrm{g})\left(\mathrm{mol} . \mathrm{L}^{-1}\right)^{-1^{1 / n}}\right) ; \mathrm{C}_{\mathrm{e}}, \quad$ la concentration du soluté à l'équilibre (mol.L' $\left.{ }^{1}\right)$.

Les paramètres de Freundlich $\mathrm{K}_{\mathrm{F}}$ et $\mathrm{n}$, et, le facteur de corrélation spécifique ou global $\left(\mathrm{R}^{2}\right.$ ou $\left.\mathrm{R}^{2} \mathrm{~g}\right)$ sont présentés dans le Tableau 11. Les coefficients de corrélation déterminés montrent que l'adsorption de QBC sur CAP BP suit avec satisfaction le modèle de Freundlich et suppose une adsorption sur une surface hétérogène ; ce qui est en accord avec la surface du charbon actif représentée par l'image SEM (Figure 2) qui montre une certaine hétérogénéité de la surface du charbon actif.

Les paramètres $\mathrm{K}_{\mathrm{F}}$ sont très élevés $\left(0,590.10^{3}\right.$ à $\left.12,558 \cdot 10^{3}\right)$ pour tous les systèmes et indiquent une capacité d'adsorption élevée des CAP BP. On observe une diminution de la capacité d'adsorption avec l'augmentation du $\mathrm{pH}$.

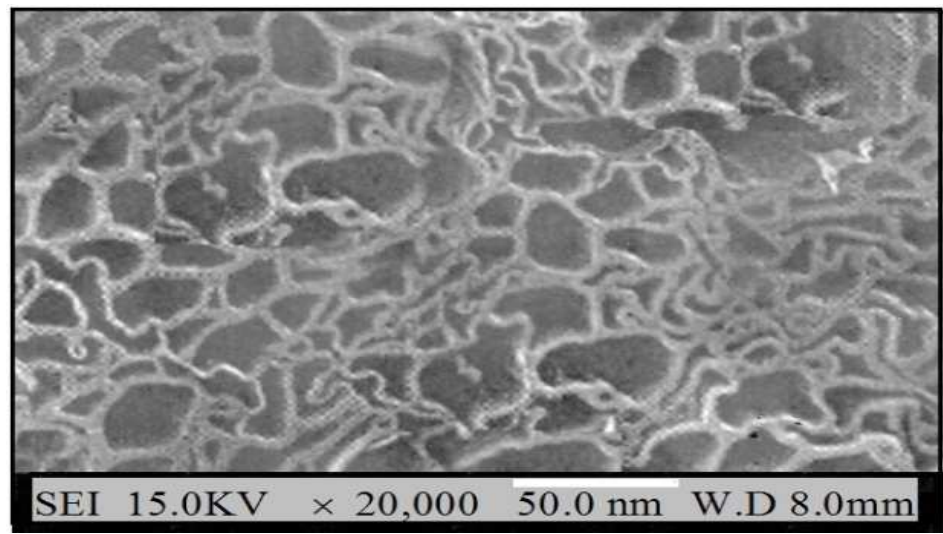

Figure 2 : Cliché SEM de la structure du charbon actif BP. 


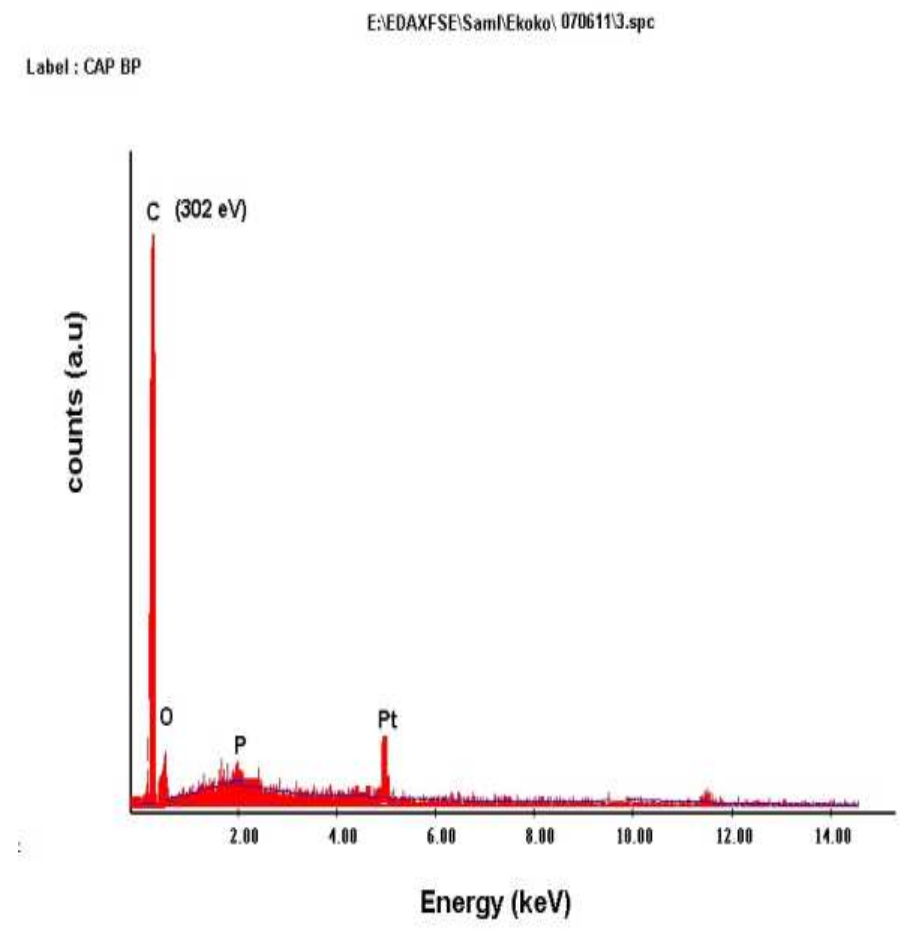

Figure 3: Spectre EDS du CAP BP.

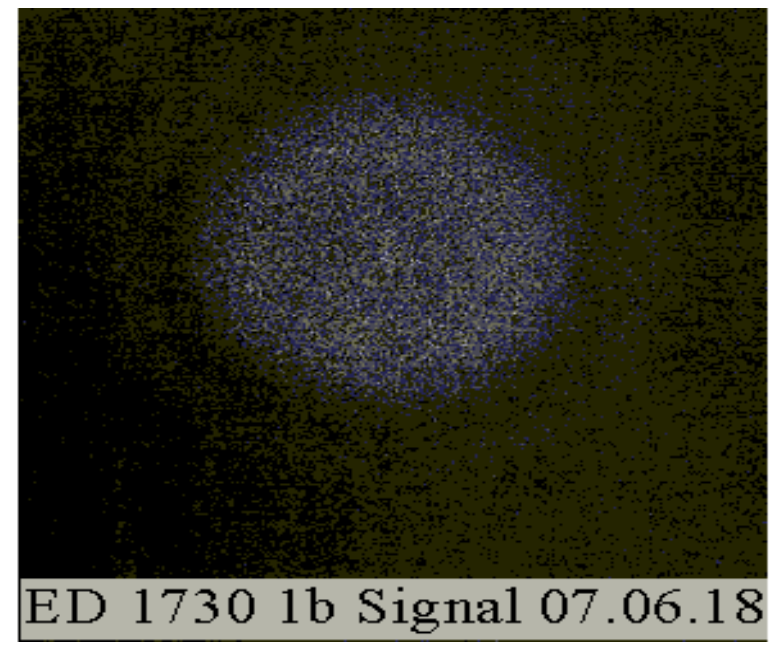

Figure 4: Image TEM du CAP BP. 


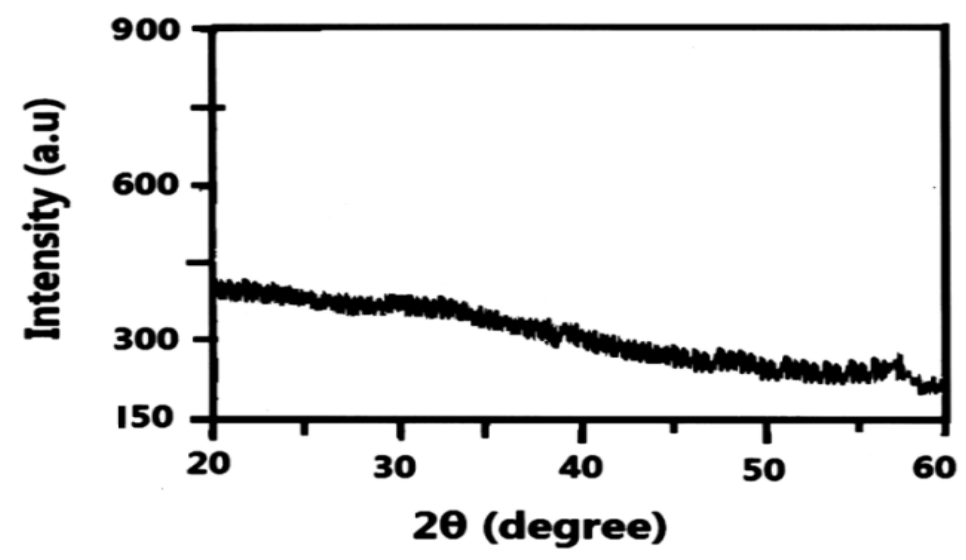

Figure 5 : Spectre XRD du CAP BP.

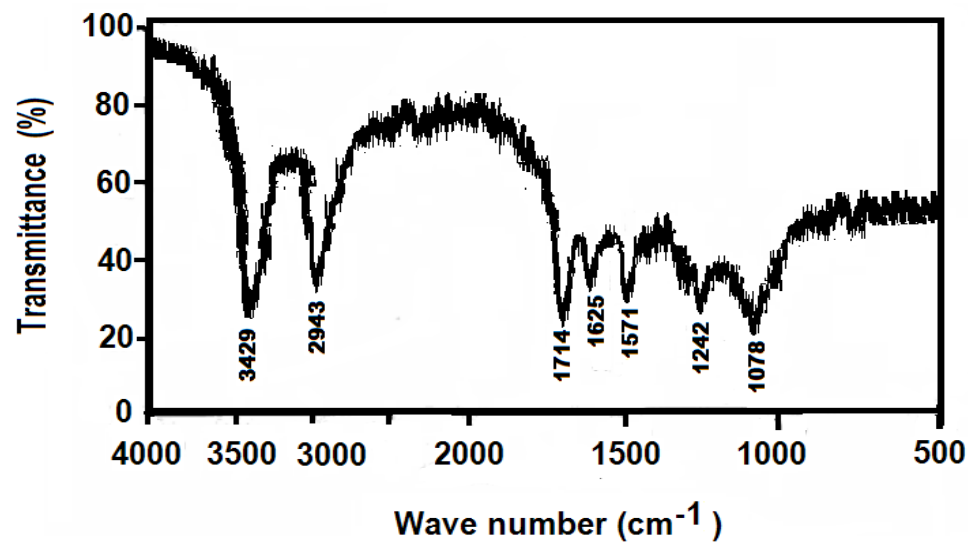

Figure 6: CAP BP- infrarouge.

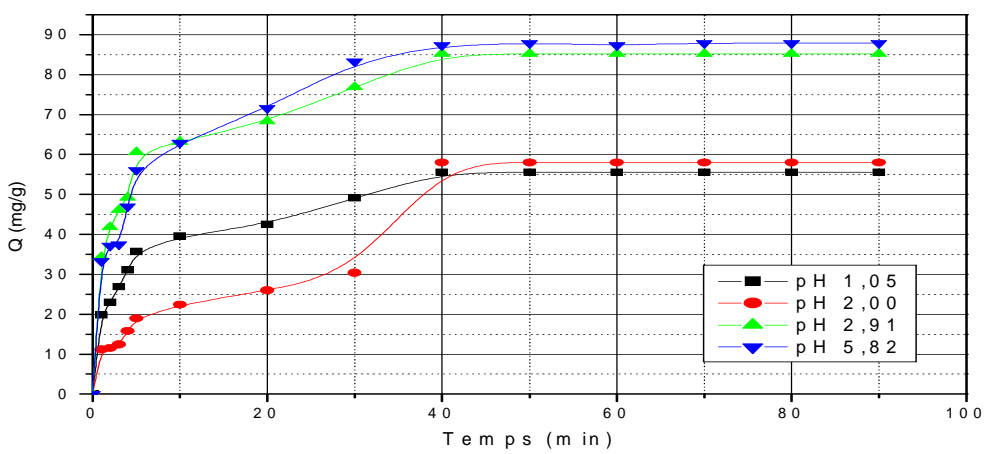

Figure 7 : Capacité d'adsorption $(\mathrm{Q})$ du charbon actif $\mathrm{BP}$ vis-à-vis de $\mathrm{QBC}$ en fonction du temps et du $\mathrm{pH} ; \mathrm{C}_{\mathrm{i}}: 28,00 \mu \mathrm{mol} . \mathrm{L}^{-1} ; \mathrm{m}_{\mathrm{C}}: 1 \mathrm{mg} ; \mathrm{V}: 50 \mathrm{~mL}$. 


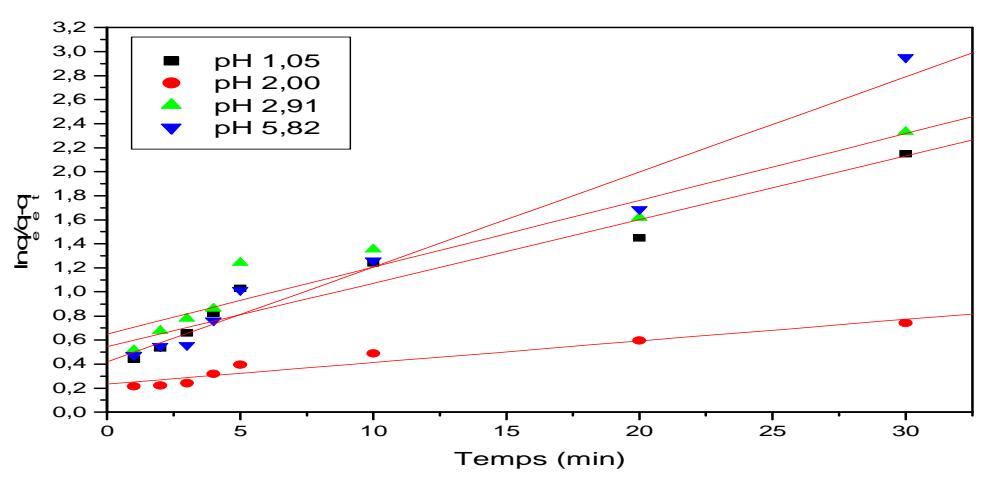

Figure 8 : Application du modèle cinétique de pseudo -ordre 1 pour l'adsorption de $\mathrm{QBC}$, en solution aqueuse à différents $\mathrm{pH}$, sur CAP BP.

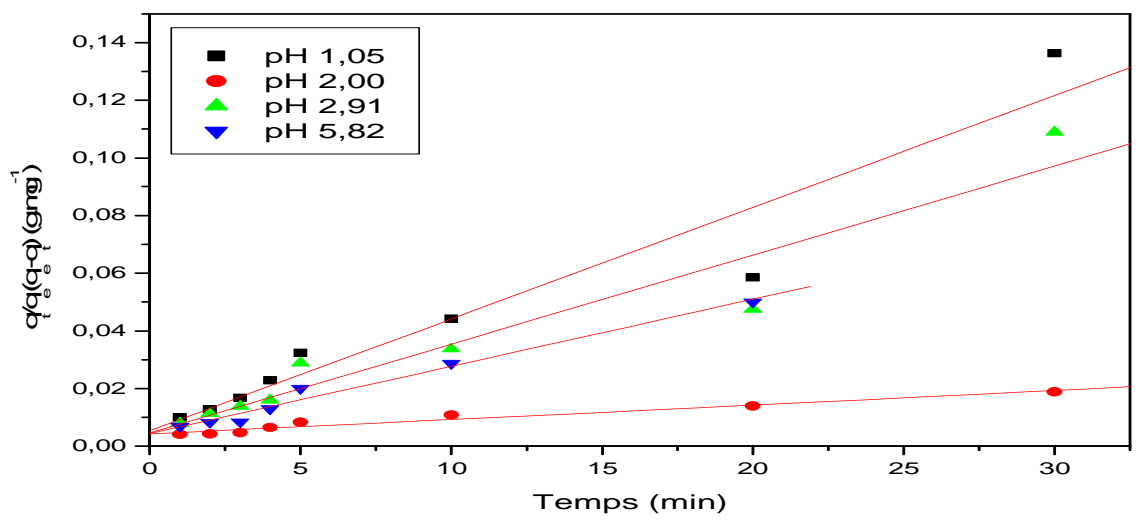

Figure 9: Application du modèle cinétique de pseudo -ordre 2 pour l'adsorption de QBC, en solution aqueuse à différents $\mathrm{pH}$, sur CAP BP.

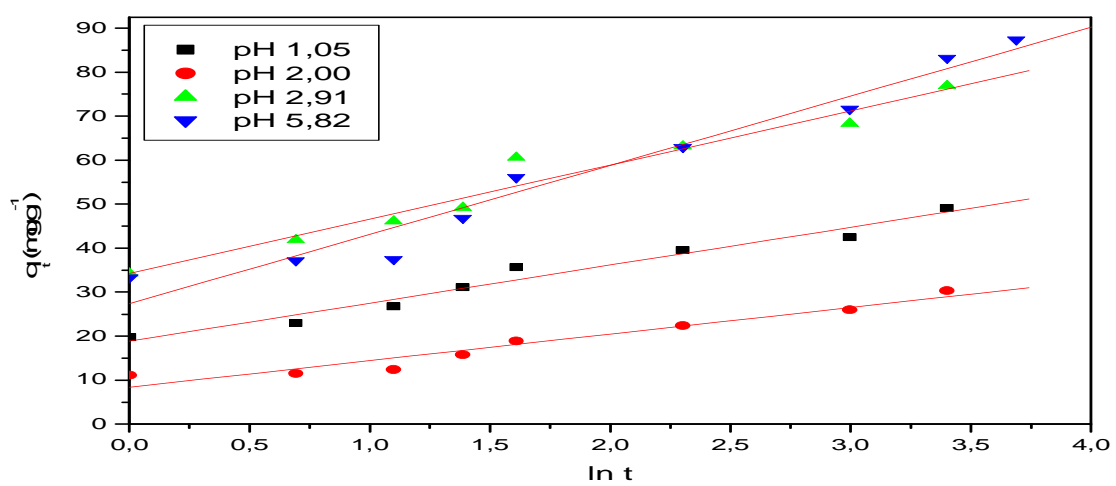

Figure 10: Application du modèle cinétique d'ELOVICH pour l'adsorption de QBC, en solution aqueuse à différents $\mathrm{pH}$, sur charbon actif $\mathrm{BP}$. 


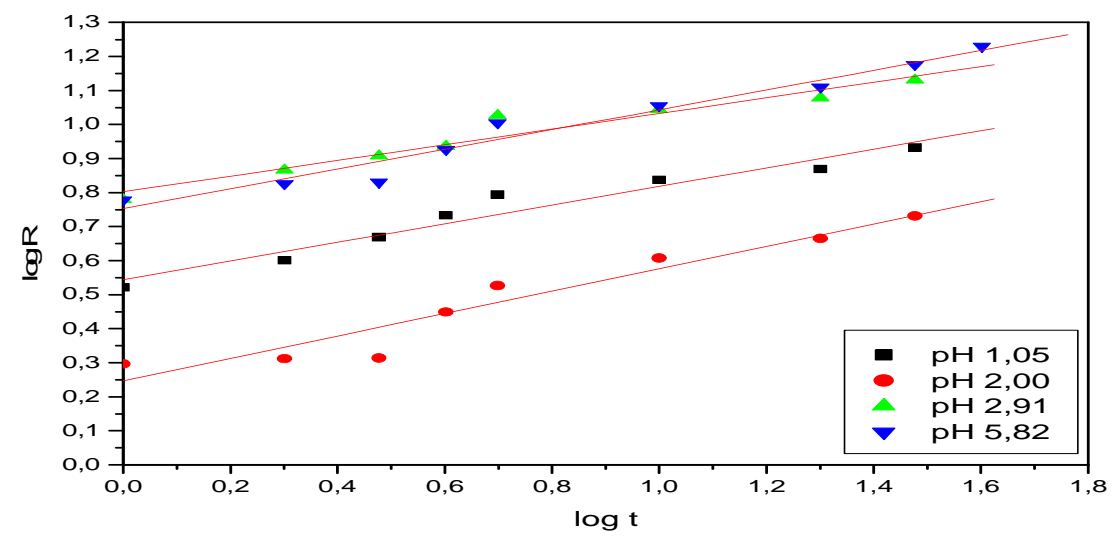

Figure 11: Application du modèle cinétique de diffusion intraparticulaire pour l'adsorption de QBC, en solution aqueuse à différents $\mathrm{pH}$, sur charbon actif BP.

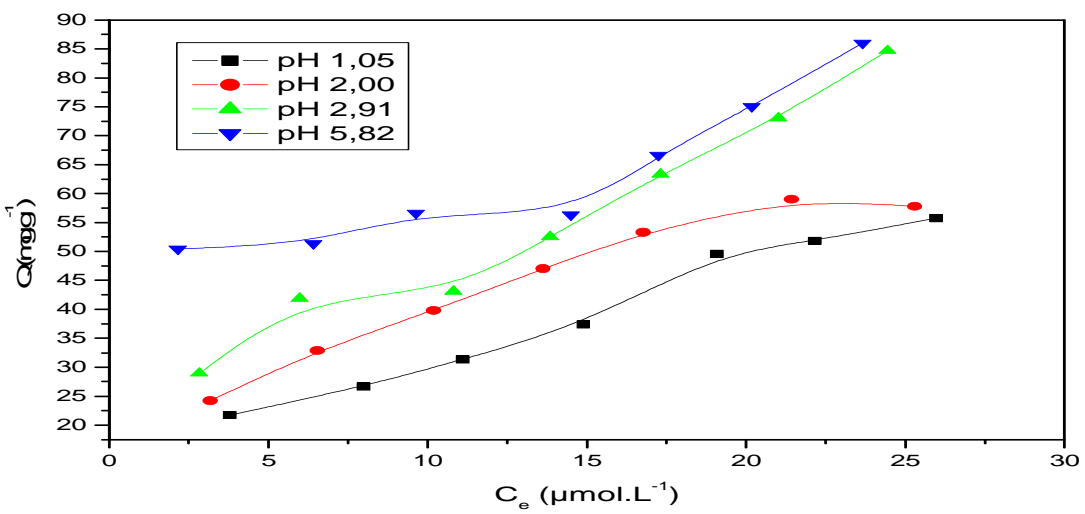

Figure 12: Isothermes d'adsorption de $\mathrm{QBC}$ sur charbon actif $\mathrm{BP}$, en solution aqueuse à différents $\mathrm{pH} ; \mathrm{m}_{\mathrm{C}}: 1 \mathrm{~g} ; \mathrm{V}: 50 \mathrm{~mL}$.

Tableau 1 : Pouvoir adsorbant (PA) des charbons BP, B0 et des charbons commerciaux.

\begin{tabular}{lcc}
\hline Charbon & PA $_{\mathbf{B M}}\left(\mathbf{m g . g ^ { - 1 }}\right)$ & PA $_{\mathbf{~ I 2}}\left(\mathbf{m g}_{\mathbf{g}} \mathbf{g}^{\mathbf{- 1}}\right)$ \\
\hline BP & 436,94 & 917 \\
B0 & 7,19 & 471 \\
CECA & 317,29 & 765 \\
MERCK & 175,27 & 778 \\
\hline
\end{tabular}


Tableau 2: Concentration (méq. ${ }^{-1}$ ) des fonctions chimiques de surface de nature acide ou basique du charbon actif BP et MERCK (CM).

\begin{tabular}{lccccc}
\hline Charbon actif & Carboxylique & Lactone & Phénolique & \multicolumn{2}{c}{ Surface totale } \\
\cline { 5 - 6 } & & & & Acide & Basique \\
\hline BP & 4,25 & 1,15 & 0,95 & 6,35 & 0,20 \\
CM & 2,85 & 1,45 & 0,95 & 5,25 & 0,40 \\
\hline
\end{tabular}

Tableau 3 : Attributions des pics et bandes d'adsorption IR du charbon actif BP.

\begin{tabular}{lc}
\hline BP & Attributions \\
\hline 3429 & Bended OH \\
2943 & Ge cristal \\
1714 & -COOH, -COO- (lactone), dicétone \\
1625 & Quinones, ion radical \\
1571 & Cétone, esters, céto-énol \\
1078 & - COOH \\
$1800-1500$ & $-\mathrm{CO}$ - (cétone) \\
$1300-1200$ & - OH \\
$1230-1100$ & $-O H$ (phénol) \\
$<\quad 1588$ & Noyau aromatique \\
\hline
\end{tabular}

Tableau 4 : Résultats de tests de conformité aux exigences de la pharmacopée pour les charbons actifs BP et MERCK (CM).

\begin{tabular}{lccc}
\hline Paramètres & Normes & BP & CM \\
\hline Perte à la dessiccation $(\%)$ & $<15 \%$ & 1,8 & 10 \\
Résidus à la calcination & $<4 \%$ & 1,6 & 1,1 \\
Substances solubles dans & & & \\
les acides (mg) & $<35 \mathrm{mg}$ & 17,0 & 10 \\
Pouvoir décolorant $(\mathrm{mL})$ & $\Delta \mathrm{V}>0,7 \mathrm{~mL}$ & 8,5 & 9,1 \\
& & & \\
\hline
\end{tabular}

Tableau 5: Concentrations à l'équilibre $\left(\mathrm{C}_{\mathrm{e}}\right)$, capacités ultimes $\left(\mathrm{Q}_{\mathrm{m}}\right)$ d'adsorption et chaleurs d'adsorption directes $\left(\mathrm{Q}_{\mathrm{ads}}\right)$ de la Quinine Bichlorhydrate $(\mathrm{QBC})$ sur charbon actif $\mathrm{BP} ; \mathrm{C}_{\mathrm{i}}: 28,00 \mu \mathrm{mol} . \mathrm{L}^{-1} ; \mathrm{m}_{\mathrm{C}}: 1 \mathrm{mg} ; \mathrm{V}: 50 \mathrm{~mL}$.

\begin{tabular}{|c|c|c|c|}
\hline pH & $\mathrm{C}_{\mathrm{e}}\left(\mu \mathrm{mol} . \mathrm{L}^{-1}\right)$ & $Q_{m}\left(\mathrm{mg} \cdot \mathrm{g}^{-1}\right)$ & $-Q_{\text {ads }}\left(\mathrm{kJ} \cdot \mathrm{mol}^{-1}\right)$ \\
\hline 1,05 & 25,98 & 55,55 & 26,483 \\
\hline 2,00 & 25,38 & 58,00 & 26,542 \\
\hline 2,91 & 24,42 & 85,18 & 26,639 \\
\hline 5,82 & 23,50 & 87,87 & 26,735 \\
\hline
\end{tabular}


Tableau 6 : Paramètres cinétiques de la réaction de surface de pseudo-ordre 1 et 2 pour l'adsorption de la Quinine Bichlorhydrate (QBC) sur charbon actif BP.

\begin{tabular}{ccccccl}
\hline $\mathbf{p H}$ & \multicolumn{2}{c}{ Pseudo-ordre $\mathbf{1}$} & \multicolumn{3}{c}{ Pseudo-ordre 2 } \\
\cline { 2 - 7 } & $\mathbf{k}_{\mathbf{1}}$ & $\mathbf{R}^{\mathbf{2}}$ & $\mathbf{R}^{\mathbf{2}} \mathbf{g}$ & $\mathbf{k}_{\mathbf{2}}$ & $\mathbf{R}^{\mathbf{2}}$ & $\mathbf{R}^{\mathbf{2}} \mathbf{g}$ \\
\hline 1,05 & 0,053 & 0,9352 & 0,9388 & 0,0039 & 0,9285 & 0,9476 \\
2,00 & 0,018 & 0,9313 & & 0,0005 & 0,9606 & \\
2,91 & 0,056 & 0,9255 & & 0,0031 & 0,9204 & \\
5,82 & 0,079 & 0,9632 & & 0,0023 & 0,9811 & \\
\hline
\end{tabular}

Tableau 7: Paramètres cinétiques d'ELOVICH pour l'adsorption de la Quinine Bichlorhydrate (QBC) sur charbon actif BP.

\begin{tabular}{ccccc}
\hline $\mathbf{p H}$ & $\boldsymbol{\alpha}\left(\mathbf{m g} \cdot \mathbf{g}^{-\mathbf{1}} \cdot \mathbf{m i n}^{-\mathbf{1}}\right)$ & $\boldsymbol{\beta}\left(\mathbf{g} \cdot \mathbf{m g}^{-\mathbf{1}}\right)$ & $\mathbf{R}^{\mathbf{2}}$ & $\mathbf{R}^{\mathbf{2}} \mathbf{g}$ \\
\hline 1,05 & 77,009 & 0,1159 & 0,9698 & 0,9562 \\
2,00 & 24,208 & 0,1656 & 0,9466 & \\
2,91 & 199,178 & 0,0881 & 0,9593 & \\
5,82 & 89,912 & 0,0637 & 0,9491 & \\
\hline
\end{tabular}

Tableau 8: Paramètres cinétiques de diffusion intraparticulaire pour l'adsorption de la Quinine Bichlorhydrate (QBC) sur charbon actif BP.

\begin{tabular}{|c|c|c|c|c|}
\hline $\mathbf{p H}$ & $\mathrm{k}_{\mathrm{di}}\left(\mathrm{mg} \mathrm{g}^{-1} \cdot \mathrm{min}^{-1}\right)$ & $\mathbf{a}$ & $\mathbf{R}^{2}$ & $\mathbf{R}^{2} g$ \\
\hline 1,05 & 3,5006 & 0,2736 & 0,9466 & 0,9809 \\
\hline 2,00 & 1,7637 & 0,3294 & 0,9662 & \\
\hline 2,91 & 6,3447 & 0,2300 & 0,9523 & \\
\hline 5,82 & 5,6982 & 0,2843 & 0,9766 & \\
\hline
\end{tabular}

Tableau 9: Paramètres de Langmuir pour l'adsorption de QBC sur charbon actif, à différents $\mathrm{pH}$.

\begin{tabular}{|c|c|c|c|c|}
\hline pH & $Q_{m}\left(\mathrm{mg} \mathrm{g}^{-1}\right)$ & $\mathrm{K}_{\mathrm{L}} \cdot \mathbf{1 0}^{-5}\left({\left.\mathrm{~L} . \mathrm{mol}^{-1}\right)}^{-1}\right.$ & $\mathbf{R}^{2}$ & $\mathbf{R}^{2} \mathbf{g}$ \\
\hline 1,05 & 110,18 & 0,383 & 0,9714 & 0,9299 \\
\hline 2,00 & 110,21 & 0,611 & 0,9168 & \\
\hline 2,91 & 94,73 & 1,144 & 0,7390 & \\
\hline 5,82 & 90,02 & 1,902 & 0,7303 & \\
\hline
\end{tabular}


Tableau 10: Paramètre d'équilibre $\left(\mathrm{R}_{\mathrm{L}}\right)$ pour l'adsorption de $\mathrm{QBC}$ sur charbon actif, à différents $\mathrm{pH}$.

\begin{tabular}{cc}
\hline $\mathbf{p H}$ & Paramètre $\mathbf{R}_{\mathbf{L}}$ \\
\hline 1,05 & $0,4825-0,8671$ \\
2,00 & $0,3689-0,8036$ \\
2,91 & $0,2379-0,6860$ \\
5,82 & $0,1581-0,5679$ \\
\hline
\end{tabular}

Tableau 11: Paramètres de Freundlich pour l'adsorption de QBC sur charbon actif BP, à différents $\mathrm{pH}$.

\begin{tabular}{ccccc}
\hline $\mathbf{p H}$ & $\begin{array}{c}\mathbf{K}_{\mathbf{F}} \cdot \mathbf{1 0}^{-\mathbf{3}} \\
\left((\mathrm{mg} / \mathrm{g})\left(\mathrm{mol} \cdot \mathrm{L}^{-1}\right)^{-1 / n}\right)\end{array}$ & $\mathbf{1 / n}$ & $\mathbf{R}^{\mathbf{2}}$ & $\mathbf{R}^{\mathbf{2}} \mathbf{g}$ \\
\hline 1,05 & 12,558 & 0,5167 & 0,9747 & 0,9450 \\
2,00 & 10,539 & 0,4822 & 0,9957 & \\
2,91 & 8,087 & 0,4439 & 0,9142 & \\
5,82 & 0,590 & 0,1969 & 0,6620 & \\
\hline
\end{tabular}

\section{DISCUSSION}

L'objectif de ce travail était de préparer le charbon actif par imprégnation de la Bagasse de canne à sucre dans une solution de $\mathrm{H}_{3} \mathrm{PO}_{4}$ et d'étudier l'adsorption de la quinine bichlorhydrate sur ce charbon. Les résultats obtenus sur la préparation et la caractérisation du charbon actif suggèrent que l'imprégnation chimique permet le développement de la microporosité responsable de la réaction chimique de surface. La différence de niveau d'adsorption observée entre le BM (grosse molécule ne se fixant que dans les maxi et moyens micropores) et l'iode (petite molécule pouvant se fixer jusqu'aux minimicropores) (Alvarez-Merino, 2005 ; Stavropoulos, 2005) suggère que le processus d'imprégnation développe davantage les maxi micropores et les moyens micropores. L'absence des composés non carbonisés et la teneur élevée en carbone dans le charbon actif BP indiquent une bonne carbonisation du matériau précurseur. Le matériau préparé est donc essentiellement constitué du carbone. La teneur élevée en oxygène du charbon actif BP suggère une forte composition de ce charbon actif en groupements fonctionnels oxygénés de surface.

Le faible taux en cendres obtenu indique une matrice organique importante. Les cendres peuvent être constituées des silicates, des aluminosilicates, des oxydes de calcium, magnésium, fer, potassium et sodium et sont susceptibles de contribuer dans les propriétés catalytiques du charbon actif (Osnick, 2009).

L'imprégnation avec l'acide phosphorique favorise plus la formation des groupements acides carboxyliques que les fonctions acides de type lactone ou phénol. La proportion élevée des fonctions acides de surface du charbon actif BP confère à ce charbon actif une polarité et une hydrophilie élevée et augmente la densité de charge négative superficielle, comme l'indiquent Maurya et al. (2008) pour d'autres adsorbants. L'oxydation du carbone conduisant à la formation des groupements oxygénés a lieu 
principalement au cours du processus d'activation. Le groupe hydroxyle se forme en premier à partir du groupe $-\mathrm{CH}$. Ensuite, le groupe hydroxyle s'oxyde en groupe carbonyle puis en groupe carboxylique (Mangun et al ,1999). La séquence oxydative est donc la suivante :

$$
-\mathrm{CH} \rightarrow-\mathrm{C}-\mathrm{OH} \rightarrow-\mathrm{CO}-\rightarrow-\mathrm{COOH}
$$

La nature et la teneur des groupements fonctionnels formés à la surface du charbon actif dépendent de la nature du matériau précurseur, de l'agent d'imprégnation utilisé et du mode de préparation du charbon actif.

L'absence des métaux lourds dans ce charbon actif exclut l'intoxication par les métaux lourds lors de l'utilisation médicinale de ce charbon. A la lumière des résultats obtenus, il ressort que le charbon actif $\mathrm{BP}$ préparé dans cette étude est conforme aux normes de la pharmacopée, comme c'est aussi le cas pour le charbon actif MERCK.

Les valeurs relativement élevées de $\mathrm{Q}_{\mathrm{ads}}$ sont dues aux interactions entre les fonctions de surface du CAP et la charge du soluté, QBC, et aussi aux interactions $\pi-\pi$ de la structure du graphène (donneur d'électrons) et celle des noyaux aromatiques de la structure quinoléique de la quinine bichlorhydrate (Accepteur d'électrons).

Les modèles de diffusion intraparticulaire $\left(\mathrm{R}^{2} \mathrm{~g} \quad 0,9809\right)$ et d'Elovich $\left(\mathrm{R}^{2} \mathrm{~g}\right.$ 0,9562) décrivent mieux l'adsorption de QBC sur le CAP BP, ce qui suppose que l'adsorption de QBC sur le CAP BP est déterminée par la diffusion intraparticulaire des molécules du soluté à travers les macropores, les mésopores et les micropores, mais aussi par la fixation des molécules de QBC sur la surface ou les groupements fonctionnels $\mathrm{du}$ charbon actif. Dans les courbes cinétiques exprimant $\mathrm{Q}$ en fonction $\mathrm{du}$ temps (t) (Figure 7), la première partie de la courbe (ascendante) est attribuée à la diffusion du soluté de la solution à la surface de l'adsorbant. La seconde portion (en arc) indique l'étape où la diffusion intraparticulaire est limitante de la vitesse, et enfin la troisième portion est attribuée à l'état d'équilibre final où la diffusion intraparticulaire tend à s'annuler. La forme des courbes cinétiques obtenues avec les modèles que nous avons développés permet de dire si l'adsorption est mieux décrite par le modèle de pseudo-ordre 1 ou de pseudo-ordre 2. L'observation des courbes présentées par les Figures 8 et 9 montre que les courbes tendent vers zéro aux faibles temps pour le modèle cinétique d'ordre 2 et non pour le modèle cinétique d'ordre 1 . Le modèle cinétique de pseudo-ordre 2 décrit donc mieux l'adsorption de QBC sur charbon actif BP comparé au modèle cinétique de pseudo-ordre 1 ; ceci est en accord avec les coefficients de corrélation déterminés pour ces deux modèles (Tableau 6).

L'isotherme de type $\mathrm{L}$ pour l'adsorption de QBC sur BP à $\mathrm{pH}$ 2,00 correspond aux isothermes dites de «Langmuir ». Cette isotherme présente aux faibles concentrations une concavité tournée vers le bas qui indique une saturation progressive des sites d'adsorption. Ce qui montre que les interactions entre les particules de QBC adsorbées sont faibles et donc que les molécules de soluté s'adsorbent préférentiellement horizontalement. La compétition d'adsorption entre les molécules de QBC et le solvant est faible. Le plateau observé aux concentrations élevées indique la saturation des sites d'adsorption aux concentrations élevées. La saturation des sites d'adsorption indique un recouvrement total de la surface du charbon actif. La pente initiale de l'isotherme $\mathrm{L}$ est faible, ce qui indique que les molécules de QBC exhibent une faible affinité pour le charbon actif (Kifuani et al., 2004). Ces résultats sont en accord avec ceux d'autres Auteurs qui étudient l'adsorption d'autres molécules organiques sur d'autres adsorbants (Sun et al., 2012).

La corrélation avec le modèle de Langmuir (Edwin, 2008 ; Matos et al., 2011) suppose que :

- chaque site du charbon actif ne peut être occupé que par une molécule, d'où, la formation d'une couche monomoléculaire à la surface du charbon actif ; 
- tous les sites d'adsorption sont équivalents et donc isoénergétiques ;

- les interactions entre les molécules adsorbées sur la surface sont nulles ou négligeables.

Le paramètre $\mathrm{R}_{\mathrm{L}}$ indique si l'adsorption est non favorable $\left(R_{L}>1\right)$, linéaire $\left(R_{L}=1\right)$, favorable $\left(0<\mathrm{R}_{\mathrm{L}}<1\right)$ ou irréversible $\left(\mathrm{R}_{\mathrm{L}}=0\right)$ (Karthikeyan et al., 2005). Les valeurs du paramètre d'équilibre inférieures à 1 obtenues (Tableau 10) indiquent que l'adsorption de QBC sur le CAP BP est favorable, pour tous les $\mathrm{pH}$ étudiés.

Les valeurs élevées de $\mathrm{K}_{\mathrm{F}}$ indiquent que les molécules de QBC possèdent une forte affinité vis-à-vis des CAP BP. Les valeurs de $1 / n$ sont situées entre 0 et 1 et indiquent que l'adsorption est favorable et correspond à un processus d'adsorption chimique (Ho et Mc Kay, 1998). Ceci est en accord avec les fortes énergies d'adsorption rapportées par les paramètres $\mathrm{K}_{\mathrm{L}}$ obtenus avec le modèle de Langmuir. L'adsorption de QBC se fait donc selon un mécanisme intermédiaire entre physique et chimique.

\section{Conclusion}

L'objectif de cette étude était de préparer le charbon actif avec la Bagasse de canne à sucre (BP), de le caractériser et d'évaluer sa capacité à adsorber la Quinine Bichlorhydrate (QBC). Le charbon actif obtenu possède une porosité très développée observable par l'image SEM et une surface spécifique très grande $\left(1439,46 \mathrm{~m}^{2} / \mathrm{g}\right)$. Ce charbon actif est acide ( $\mathrm{pH}$ contact 6,2$)$ et son $\mathrm{pH}_{\mathrm{ZPC}}$ est de 6,2. L'imprégnation avec l'acide phosphorique développe davantage les fonctions acides carboxyliques comparées aux fonctions lactone ou phénol. La cinétique d'adsorption de la Quinine Bichlorhydrate sur le charbon actif a été étudiée à l'aide de quatre modèles cinétiques. Les résultats obtenus montrent que les modèles cinétiques de diffusion intraparticulaire et d'Elovich décrivent mieux l'adsorption de QBC sur le charbon actif BP. Les isothermes d'adsorption sont essentiellement du type $\mathrm{S}$ pour tous les
$\mathrm{pH}(1,05,2,91$ et 5,82$)$, sauf à $\mathrm{pH} 2,00$ où l'on a une isotherme de type L. Les modèles de Freundlich et de Langmuir sont appropriés pour décrire l'adsorption de QBC sur charbon actif BP. L'adsorption de QBC sur charbon actif BP est essentiellement physique. Le paramètre d'équilibre $R_{L}$ et le paramètre $1 / n$ indiquent que l'adsorption de QBC sur le charbon actif BP est favorable. Le charbon actif BP est donc indiqué pour l'élimination de la Quinine Bichlorhydrate, en cas d'intoxication.

\section{REMERCIEMENTS}

Nous remercions sincèrement les Professeurs ZHU et JIN (Shanghai University) et Docteur XIN LIHUI (The National Center of Shangai Institute of measurement and testing technology) pour les analyses SEM, EDS, TEM et XRD effectuées dans leurs laboratoires.

\section{REFERENCES}

Adamson AW. 1982. Physical Chemistry of Surfaces $\left(4^{\text {th }}\right.$ edn). John Wiley and Sons: New York.

Alvarez-Merino M, Lopez-Ramon V, Moreno-Castilla C. 2005. A study of static and dynamic adsorption of $\mathrm{Zn}$ (II) ions on carbon materials from aqueous solutions. Journal of colloid and Interface Science, 288: 335-341.

Arivoli S, Prasath PMD, Thenkuzhali M. 2007. Adsorption of chromium ion by acid activated low cost carbon. EJEAFCHE, 6(9): 2323-2340.

Avom J, Ketcha M, Matip MRL et Germain P. 2001. Adsorption de l'acide acétique par des charbons d'origine végétale. African Journal of Science and Technology, Science and Engineering Series, 2(2): 1-7.

Balci S, Dogu T, Yucel H. 1994. Characterization of activated carbons produced from Almond Shell and Hazelnut Sell. J. Chem. Techn. Biotechnol., 60: 419-426. 
Boehm. 1966. Chemical Identification of Surface Groups. Academic Press: 179274.

Bouchemal F, Achour S. 2007. Essais d'adsorption de la tyrosine sur charbon actif en grains et en poudre. Larhyss Journal, 6: 81-89.

Chien SH, Clayton WR. 1980. Application of Elovich equation to the kinetics of phosphate release and sorption on soils. Soil Sci. Soc. Am. J., 44: 265-268.

Edwin Vasu A. 2008. Surface modification of activated carbon for enhancement of Nickel (II) adsorption. E-Journal of chemistry, 5(4): 814-819.

Hafiz HS, Craig LP, George AS, Sinha R, Radha K. 2011. The fate and transport of the $\mathrm{SiO}_{2}$ nanoparticles in a granular activated carbon bed and their impact on the removal of VOCs. J. Hazard. Mater., 193: 95-101.

Ho YS, Mc Kay G. 1998. Kinetics models for the sorption of dye from aqueous solution by wood. Process Safety Environmental Protection, 76: 183-191.

Ho YS, Mc Kay G. 2000. The kinetics of sorption of divalent metal ions onto sphagnum moss peat. Water Research, 34(3): 735-742.

Khartikeyan G, Anita P, Alagumuthu G. 2005. Fluoride adsorption studies of montmorrillonite clay. Ind. J. Chem. Technol., 12: 263-272.

Khelifi A, Temdrara L, Addoun A. 2009. Effet de la texture poreuse et de la structure chimique sur l'adsorption du bleu de méthylène par des charbons actifs oxydés. J. Soc. Alger. Chim., 19(1): 1325.

Kifuani KM, Mukana WM, Noki V, Musibono EA, Nzuzi P, Panzu P, Kunyima B. 2004. Adsorption de bleu de méthylène en solution aqueuse sur charbon actif obtenu à partir des sciures végétales : Préparation et caractérisation du charbon actif. Rev. Cong. Sci. Nucl., 20(1-2): 215224.
Kumar A, Kumar S, Gupta DV. 2007. Adsorption of phenol and 4-nitrophenol on granular activated carbon in basal salt medium: Equilibrium and kinetics. Hazardous Materials, 147: 155-166.

Laximi GS, Ahmazzaman MD. 2010. Adsorption technique for the removal of phenolic compounds from wastewater using low-cost natural adsorbents. Assam University Journal of Science and Technology, 5(2): 156-166.

Li L. 2002. Effects of activated surface chemistry and pore structure on the adsorption of trace organic contaminants from aqueous solution. Ph.D. Thesis, North Carolina State University, North Carolina, p.26.

Li Y, Du Q, Wang X, Zhang P, Wang D, Wang Z. 2010. Removal of lead from aqueous solution by activated carbon prepared from Enteromorpha prolifera by zinc chloride activation. J. Hazard. Mater., 183(1-3): 583-589.

Lin C-L, Cheng Y-H, Liu Z-S, Chen J-J. 2011. Metal catalysts supported on activated carbon fibers for removal of polycyclic aromatic hydrocarbons from incineration flue gas. J. Hazard. Mater, 197: 254-263.

Mangun CL, Benak KR, Daley MA, Economy J. 1999. Oxidation of activated carbon fibers: effect on pore size, surface chemistry, and adsorption properties. Chem Mater, 11: 3476-3483.

Maria RJ, Arana R, Mazzoco RR. 2010. Adsorption studies of methylene blue and phenol onto black stone cherries prepared by chemical activation. J. Hazard. Mater., 180(1-3): 656-661.

Matos J, Nahas C, Rojas L, Rosales M. 2011. Synthesis and characterization of activated carbon from sawdust of Algarroba wood: 1. Physical activation and pyrolysis. J. Hazard. Mater, 196: 360-369.

Maurya MS, Mittal AK, Correl P. 2008. Evaluation of adsorption potential of adsorbents: A case of uptake of cationic 
dyes. Journal of Environmental Biology, 29(1): 31-36.

Muhammad JI, Muhammad NA. 2007. Adsorption of dyes from aqueous solutions on activated charcoal. $J$. Hazard. Mater., 39: 57-66.

Osnick J. 2009. Etude du potentiel d'utilisation de résidus agricoles haïtiens pour le traitement par biosorption d'effluents pollués. Thèse de doctorat, Sciences de l'Environnement industriel et Urbain, INSA, Lyon, p.130.

Padhye L, Wang P, Karanfil T, Huang C-H. 2010. Unexpected role of activated carbon in promoting transformation of secondary amines to n-nitrosamines. Environ. Sci. Technol., 44: 4161-4168.

Quintanilla A, Menéndez N, Tornero J, Casas JA, Rodriguez JJ. 2008. Environmental : Surface modification of carbonsupported iron catalyst during the wet air oxidation of phenol: Influence on activity, selectivity and stability. Applied Catalysis B., 81: 105-114.

Schimmel D, Fagnani KC, Olivier Santos JB, Baros SD, Antonio da Silva. 2010.
Adsorption of Turquoise Blue QG reactive dye on commercial activated carbon in batch reactor: Kinetic and equilibrium studies. Brazilian Journal of Chemical Engineering, 27(2): 289-298.

Srivastava VC, Swamy MM, Malli D, Prasad B, Mishra IM. 2006. Adsorptive removal of phenol by bagasse fly ash and activated carbon: Equilibrium, Kinetics and thermodynamics. Colloids and Surfaces A: Physicochemical and Engineering Aspects, 272: 89-104.

Stavropoulos GG. 2005. Precursor materials suitability for super activated carbons production. Fuel Processing Technology, 86: 1165-1173.

Sun L, Yu H, Fugestu B. 2012. Graphene oxide adsorption enhanced by in situ reduction with sodium hydrosulfite to remove Acridine orange from aqueous solution. J. Hazard. Mater., 203-204: 101-110.

US XXI. 1985. US Pharmacopoeial National Formular (6 ${ }^{\text {th }}$ edn). United States. 